\title{
Feedbackpraktiken im Schreibcoaching: Texte besprechen in der Hochschullehre
}

\author{
Cordula Schwarze ${ }^{1}$
}

Angenommen: 18. November 2020 / Online publiziert: 15. Dezember 2020

(c) Der/die Autor(en) 2020

\section{Zusammenfassung}

Im Mittelpunkt des Artikels stehen Feedback und Textfeedback als spezielle Interventionsformen innerhalb des Coachings von Schreib(lern)prozessen im spezifischen Setting der disziplinär-germanistischen, professionsorientierten universitären Lehre. Kompetenzorientierte schreibdidaktische Lehre und Coaching weisen Schnittmengen auf, die sich an der Orientierung am schreibenden Individuum, an individuellen Beratungsbedarfen sowie am Problembezug zeigen. Das wird im Artikel methodisch in interaktionsanalytisch-linguistischer Perspektive bearbeitet und anhand von videographierten Seminarinteraktionen wird aufgezeigt, wie diese Feedbackprozesse gestaltet sind. Dabei werden wiederkehrende diskursive Feedbackpraktiken und ihre sprachliche Realisierung herausgearbeitet, insbesondere Situationseröffnungen sowie Fragen des Umgangs mit Autorschaft. Die empirische Basis sind videographierte Gespräche im Seminar, bezeichnet als Auswertungsgespräche, die sich durch eine systematische Anwendung von Textfeedback auszeichnen. Die Resultate der Analysen zeigen, dass sich anhand der Handlungstypik in der Interaktion ein neuer Gesprächstyp zwischen Coaching und Lehre entwickelt. Das ermöglicht, praktische Implikationen für beide Settings aufzuzeigen, die für beide Seiten produktiv gemacht werden können.

Schlüsselwörter Auswertungsgespräch · Coaching $\cdot$ Feedbackpraktiken $\cdot$ Hochschullehre $\cdot$ Schreibcoaching . Wissenschaftliches Schreiben

\section{Feedback Practices in Writing Coaching: Discussing Texts in University Teaching}

\begin{abstract}
This paper focuses on feedback processes on texts, produced in writing coaching as a particular type of pedagogic intervention in the specific setting of a university's German department, in which writing is taught as part of the disciplinary, profession-oriented curriculum. There is an important overlap between competency-oriented writing instruction and coaching, which is manifested in their shared orientation towards the writing individual, individual counselling needs and a common interest in addressing specific problems. Methodologically, the study is grounded in interactional linguistics and provides a linguistic analysis of videographed interactions among students and instructors. The primary aim of this paper is to demonstrate how these feedback processes unfold as well as identifying the recurrent discursive feedback practices and their linguistic realizations, in particular situations of openings and authorship questions. The database of this study is made up of videographed in-class interactions, termed "appraisal talks", whose defining characteristic is their systematic use of feedback on students' performances. The findings demonstrate the emergence of a new type of spoken interaction which, due to its specific action type, can be situated at the interface of coaching and teaching. This study should, therefore, be of value to practitioners wishing to foster further cross-fertilization of ideas between the two settings.
\end{abstract}

Keywords Academic writing $\cdot$ Appraisal talk $\cdot$ Coaching $\cdot$ Feedback practices $\cdot$ University teaching $\cdot$ Writing coaching

Cordula Schwarze

cordula.schwarze@uibk.ac.at
1 Institut für Germanistik, Universität Innsbruck, Innrain 52a, 6020 Innsbruck, Österreich 


\section{Einführung}

Im Mittelpunkt des Beitrags stehen Feedback und Textfeedback als spezielle Interventionsformen innerhalb des Coachings von Schreib(lern)prozessen in einem spezifischen Setting, der disziplinär-germanistischen, professionsorientierten universitären Lehre. Textfeedback wird dort im Rahmen sogenannter „Auswertungsgespräche“ gegeben, die die Datenbasis dieses Beitrags bilden.

Im Kontext von Lehren und Lernen wird selten von Coaching gesprochen, aber die Analogie liegt auf der Hand: Coaching wird als ein primär personen- und prozessorientiertes Beratungsformat, das „eine zielorientierte, systematische und zeitlich begrenzte Unterstützung eines Entwicklungs- und Veränderungsprozesses" (Jautz 2018, S. 34) bietet, verstanden. In Coaching- und Beratungsprozessen soll daher die Handlungsfähigkeit von Personen hergestellt werden, das ist auch Ziel kompetenzorientierter Hochschullehre. Schreibcoaching als Spezialfall von Coaching zielt darauf, individuelle Schreibprozesse zu optimieren, indem die eigenen Kompetenzen erkannt werden und spezifische Handlungsfähigkeit hergestellt wird. Das gilt auch für kompetenzorientierte Hochschullehre. Konzeptuell weisen also kompetenzorientierte schreibdidaktische Lehre (z. B. Kruse und Chitez 2012; Schmölzer-Eibinger et al. 2018; Schwarze und Rieder 2014) und Coaching Schnittmengen auf, die sich an der Orientierung am (schreibenden) Individuum, an individuellen Beratungsbedarfen sowie am Problembezug zeigen.

Darüber hinaus vollziehen sich beide Aktivitäten im und durch das Gespräch. Dies, verbunden mit seinen interaktionalen Konsequenzen, wird von Arbeiten zu Coaching oder Beratung in gesprächslinguistischer Perspektive mit einem klaren Fokus auf der Prozessualität (z. B. Deplazes et al. 2018; Graf et al. 2011; Graf 2015; Jautz 2018; Pick 2017; Rettinger 2011) oder auch in Arbeiten zu Gesprächen in der Schreibberatung eines Schreibzentrums (z. B. Grieshammer 2018; Brown 2010) akzentuiert. Die Perspektive auf den gesprächshaften Vollzug von Coaching und Lehre zieht die spezifische Bestimmung der Eigenschaften eines Gesprächs nach sich, zu denen Konstitutivität, Prozessualität, Interaktivität, Methodizität und Pragmatizität gehören (Deppermann 2008, S. 8).

Ansätze aus der gesprächsanalytischen Erforschung von Coaching geben folglich einen produktiven Rahmen, um den Interaktionstyp Auswertungsgespräch in universitärer Lehre in dieser Hinsicht zu untersuchen. Die empirische Basis des Artikels legen videographierte natürliche Gespräche im Seminar, die keine genuinen Coachingprozesse sind. Vielmehr zeigen sich in den Auswertungsgesprächen als einem neuartigen Interaktionstyp im professionsorientierten Lehr-Lern-Diskurs spezifische Interaktionscharakteristika des Coachings. Interaktionsbezogen ist eine besondere
Schnittmenge zwischen Coaching und Lehre die Bearbeitung der kommunikativen Aufgabe „Ko-Konstruieren von Veränderung" (Graf 2015, S. 11). Diese Aufgabe stellt sich in den analysierten Auswertungsgesprächen, auch wenn sie anders gelöst wird: Veränderung zeigt sich in der Lehre als professionsorientiert gebundene, zielgerichtete Veränderung von Schreibkompetenz. In Auswertungsgesprächen stehen „die reflexive Bearbeitung von Konvergenzen und Divergenzen auf der Ebene der Beobachtung, Analyse und Beurteilung sowie das Aufzeigen von Handlungsalternativen anhand eines Schreibproduktes oder mündlichen rhetorischen Ereignisses eines anwesenden Lernenden durch die Seminargruppe im Mittelpunkt. Konstituierender Faktor ist das Vorliegen eines Auswertungsobjekts, auf das sich die auswertenden Handlungen beziehen lassen" (Schwarze 2015, S. 191; Überblick: Abb. 1). Es handelt sich also um eine reflexive, zeitlich nachgestellte, explizite Bearbeitung von Auswertungsobjekten wie Texten und ihrem Herstellungsprozess in einem Lehr-Lernkontext, also um handlungsentlastete, fachspezifisch geformte Analyseund Bewertungspraxis, bestehend aus Aktivitäten des Rückmeldens, Reflektierens, Kommentierens, Bewertens, Wahrnehmens, Beschreibens und Intersubjektivierens. In diesem Sinn kann das Auswertungsgespräch selbst als eine spezifische Interventionsform von Feedback gelten, da das Erkenntnispotenzial des Gesprächs als Aushandlungsprozess verschiedener Perspektiven aktiv genutzt wird.

Mit solchen Gesprächen im germanistischen Seminar werden verschiedene Bildungsziele verfolgt. Eines davon ist die Förderung fachspezifischer Textproduktionskompetenz, andere sind die Ausbildung von fachspezifischer Urteilskraft, die Erweiterung metakognitiven Wissens über das Schreiben sowie die Förderung von Analysefähigkeiten, denn Germanist*innen sollten auf einen fertigen Text sowie Texte im Entwurfsstadium eine professionelle und fachlich gesicherte Rückmeldung geben können, die Überarbeitungsprozesse auslöst. Auswertungsgespräche sind produktiv zur Erreichung der Bildungsziele gemäß der Überzeugung, ,dass das Was des Wissens durch einen entsprechenden Erkenntnisvorgang, nämlich das Wie des Reflektierens, zustande gekommen ist" (Paul 1999, S. 245).

Der Aufsatz nimmt seinen Ausgangspunkt dezidiert vom Gesprächsmaterial, d.h. von Phänomenen der Daten (zum Korpus in Abschn. 3 und 4.1.) mit zunächst interaktionstypologischem Erkenntnisinteresse. Er bewegt sich am Schnittpunkt und den Interessen mehrerer Disziplinen wie linguistische Interaktionsforschung, Coachingprozessforschung, Schreibwissenschaft und Schreibdidaktik und versucht, sie produktiv im Hinblick auf die Prozessierung von Feedback zu verbinden (Abschn. 2). Im Mittelpunkt steht methodisch in interaktionsanalytischer Perspektive die Rekonstruktion zweier Feedbackpraktiken als wiederkehrende diskursive Praktiken und ihre sprachliche Realisie- 
rung. Das ist erstens die Handlungsaufgabe des Etablierens der Reflexionsebene und der (möglichen) Perspektiven auf den Text (Abschn. 4.2.). Gezeigt wird die systematische Bearbeitung dieser Handlungsaufgabe in den Eröffnungen der Auswertungsgespräche, einem grundlegend relevanten Aspekt des gesprächsanalytischen Zugangs. Zweitens wird ein typisches Phänomen der Auswertungsgespräche - der Umgang mit der Autorschaft ${ }^{1}$ des Textes als einer im Schreiben relevanten Kategorie - in seinen interaktiven und prozessualen Kennzeichen herausgearbeitet (Abschn. 4.3.). Mit einem Fazit (Abschn. 5) endet der Beitrag.

\section{Theoretischer Rahmen: Feedback und Textfeedback}

Im Kern ist Feedback eine Information als Rückmeldung eines Akteurs/einer Akteurin auf eine zeitlich vorgängige Leistung bzw. auf Handeln, die einfach oder komplex sein kann, sich auf verschiedene Dimensionen bezieht und das Handeln der Feedbacknehmenden beeinflussen kann. Daher setzen Hattie und Timperley (2007, S. 87) die Fragen „Where am I going? How am I going? Where to next?" zentral. Dieser sowohl retrospektive als auch prospektive Zeithorizont von Rückschau und Anbahnung der Veränderung zeichnet den Feedbackprozess und eben auch den Auswertungsprozess aus. Einigkeit besteht darin, Feedback (unabhängig von der Art seiner Prozessierung) als eine wirksame Intervention zur Unterstützung von Lernprozessen anzusehen, da es zur Reflexion eigenen Handelns anregt und in Folge auch die Veränderung eigenen Handelns (ggf. auch verfestigter Handlungsmuster) unterstützt. Dazu sind metakognitive Fähigkeiten notwendig, die self-monitoring ermöglichen und die Wirksamkeit des Feedbacks beeinflussen, wozu Informativität, Kriterienbezug und der Leistungsbzw. Handlungsbezug des Feedbacks beitragen (z. B. Kluger und DeNisi 1996, S. 255; Sadler 2010, S. 536).

Für die Anwendung in der Hochschule in allgemeinpädagogischer Perspektive ist die Literatur zum Feedback umfangreich, aber recht heterogen. Es finden sich zum einen Ratgeber (z.B. Dainton 2018), die meist zahlreiche Übungsvorschläge enthalten und eine erfahrungsbasierte Perspektive einnehmen. Zum anderen werden gut begründete Vorschläge für den Einsatz von Feedback gemacht. Dabei gelten die Beteiligung aller im Feedbackprozess sowie mehrstufige Feedbackprozesse, die Auseinandersetzung mit Anforderungen und der Erwerb von Bewertungs-

\footnotetext{
1 Mit der Nutzung des Terminus „Autorschaft“ ist im Folgenden das Konzept von Autorschaft mit all seinen Facetten adressiert. Die personenbezogene Kategorie konkreter Autor*innen eines konkreten Textes als eine Facette von Autorschaft wird davon unterschieden und entsprechend sprachlich markiert.
}

kriterien als förderlich (Hartung 2017, S. 202). Ähnlich plädieren Sadler (2010, 2013) und Bloxham (2013) dafür, dass Studierende Grundkonzepte der Beurteilung und Bewertung gemeinsam insoweit lernen, dass sie sie auf die konkrete eigene Produktion anwenden können. Bloxham (2013, S. 71) unterbreitet einen Vorschlag, der auf der Forderung nach dialogischen Ansätzen basiert. Ausgehend davon, dass professionelles Urteilen am besten durch wiederholte Partizipation an Feedbackprozessen in Verbindung mit Expert*innen gelernt wird, wird für Dialoge mit Expert*innen plädiert. Das ist nur vordergründig eine Absage an Peer Feedback, denn wie Analysen von Feedbackgesprächen im Feld der Rhetorik (zum interaktiven Verzicht auf Bewertungsdominanz z. B. Mönnich 2010, S. 245) sowie anhand der vorliegenden Daten zeigen, ist damit eine bestimmte Beteiligungsweise in der Interaktion adressiert.

Feedback als Interventionsform wird jedoch fachspezifisch ausgelegt und geformt. In Bezug auf die Entwicklung und Optimierung von Schreibkompetenz kommt Feedback als Textfeedback eine besondere Bedeutung zu: „Ohne Feedback seine Schreibfähigkeiten zu verbessern, ist nahezu unmöglich“ bemerken Frank et al. (2007, S. 107). In allen prozessualen Konzeptualisierungen von Schreiben als einer komplexen Handlung - wie sie Schreibcoaching oder Hochschullehre oder Schreibberatung zugrunde liegen -, die grob die Handlungskomplexe Planen, Formulieren und Überarbeiten enthält, ist das Überarbeiten und damit der Ansatzpunkt für Reflexion, Feedback- und Auswertungsprozesse einbezogen (z.B. Baurmann 2014; Frank et al. 2007; Grieshammer et al. 2013; Ruhmann und Kruse 2014). Gefolgt wird der Grundannahme, dass Textproduktionsprozesse dann gelingen, wenn Schreibende planvoll vorgehen und sich dieses methodische Vorgehen auch auf die Revisionsprozesse erstreckt. Feedback im Schreibcoaching dient verschiedenen Zielen, dazu gehören die Unterstützung des Schrittes des Überarbeitens im Schreibprozess (z. B. durch Randkommentare nach Knorr 2012b) sowie die Förderung von analytischen und sprachreflexiven Fähigkeiten und professionsorientierter Urteilskraft. Beides gilt als essenziell, um Schreibkompetenz zu erwerben, beides wird je nach Kontext verschieden stark gewichtet. Darüber hinaus leisten Feedbackprozesse einen Beitrag zum besseren Verständnis der Fachinhalte, indem die epistemische Funktion des Schreibens gestärkt wird (Lehnen 2011, S. 24-26).

$\mathrm{Zu}$ unterscheiden ist einerseits Feedback als eine in der Organisationsform klar umrissene didaktische Interventionsform wie beispielsweise die „Textlupe“ (BeckerMrotzek und Böttcher 2014, S. 37). Andererseits geht es um die spezifische Prozessierung des Feedbacks im Gespräch als „,handlungsentlastete Sprachreflexion“ (Paul 1999, S. 4) wie in den hier zugrundeliegenden Daten als das Geben und Nehmen von Feedback in anwesenheitsbasierter, raumzeitlich ko-präsenter Interaktion. Solchermaßen 
strukturierte Feedbackprozesse führen durch das kriteriengestützte Reflektieren und Reden über das Schreiben innerhalb von Schreibausbildungsprozessen zum Erkennen der Gebrauchsnormen, der Regularitäten, der Normbrüche und Normkonflikte (Kruse und Ruhmann 2006, S. 17). Das Reden über Texte mit dem Ziel des besseren Schreibens dient demzufolge dazu, die ,unbewussten Wissensbestände über Texte und Textualität (ein ,knowing how') zu bewusstem Wissen zu transformieren, das besprechbar und bearbeitbar wird (,knowing that ')" (Nussbaumer und Sieber 1995, S. 37). Dabei wird die Gruppe, in der über Texte und Textqualität gesprochen wird, als hilfreich angesehen, denn „,[w]as mit anderen gründlich erörtert wird, lässt den Status des Beliebigen und Zufälligen deutlich hinter sich zurück" (Baurmann 2014, S. 355). Auch in der Schreibzentrumsarbeit kommt dem Gespräch über Texte, organisiert als Peer Tutoring, eine zentrale Rolle zu, denn es ermöglicht gemeinsame Wissenskonstruktion sowie Weiterentwicklung (z. B. Grieshammer 2018, S. 18).

Feedback hilft, sich vom Text zu distanzieren, es ermöglicht ungeübten Schreibenden, Perspektivenwechsel zu erlernen, während es Geübten hilft, diesen Wechsel effizient zu gestalten und den Prozess besser steuern zu können (Kruse und Ruhmann 2006, S. 17-19). Das ist die Begründung für Feedbackaktivitäten im Schreibcoaching. Derartige Feedbackprozesse werden häufig vorab kommunikationsbezogen reguliert, so werden Feedbackregeln, Feedbackthemen bzw. -wünsche sowie die zu stellenden Fragen empfohlen (z. B. Frank et al. 2007, S. 98-99; Grieshammer et al. 2013, S. 224), auf die sich das Feedback erstrecken kann. Inwieweit diese hilfreich sind, ist nur empirisch entscheidbar.

\section{Datenbasis und methodisches Vorgehen}

Die Datenbasis des Beitrags bildet das von der Autorin systematisch erhobene Korpus „Auswertungsgespräche“, dessen Daten in curricular verankerten Seminaren einer Germanistik an einem Hochschulstandort videographiert worden sind. Subthematisch sind die Seminare der Rhetorik, Schreibwissenschaft, Argumentationswissenschaft oder dem Kommunikationsdesign zuzuordnen. Dementsprechend sind die Auswertungsobjekte verschieden, es sind Reden, Debatten, Präsentationen, Texte, Entwürfe, u.v.a.m. Die Videodaten wurden vollständig über ein akademisches Jahr erhoben; es sind natürliche Daten, also solche, die nicht zu Zwecken der Erhebung und späteren Analyse hergestellt worden sind. Beteiligt waren acht Lehrpersonen, die sehr unterschiedliche Professionsbiographien aufweisen; aufgezeichnet wurde zumeist mit zwei Kameras. Die Lehrpersonen luden immer dann zur Videographie ein, wenn sie „etwas auswerteten“. Diese Bezeichnung erwies sich als robuste und von allen Lehrpersonen geteilte Ethnokategorisierung für die videographierte Aktivität und verweist so auf vorgängige Kategorisierungen des eigenen Handelns innerhalb des professionell gebundenen Interaktionswissens. Darin zeigt sich zugleich stabile Evidenz für das Vorhandensein der Ethnokategorie „Auswerten“, was genau dann zutrifft, wenn Gesprächsteilnehmer*innen ,sich expressis verbis auf die interessierenden Phänomene beziehen, also mit Ethnokategorien operieren“ (Deppermann 2008, S. 36).

Das Korpus umfasst insgesamt ca. 92h Material. Das Teilkorpus aus schreibwissenschaftlichen Lehrveranstaltungen, das die tatsächliche Datenbasis für den Aufsatz bietet ${ }^{2}$, umfasst $26 \mathrm{~h}$ bei 15 Ereignissen und drei Lehrpersonen. Ergänzend zu den Videodaten ist das ethnographische Zusatzmaterial wie z.B. unterrichtsbezogene Unterlagen, Aufgaben, Unterlagen zum theoretischen Input, Kriterienkataloge, Textvorlagen oder Texten als ausgewählten Beispiellösungen erhoben worden. Nach abgeschlossener Datenerhebung wurde außerdem eine mehrstündige Fokusgruppendiskussion mit den videographierten Lehrpersonen durchgeführt und aufgezeichnet. Das Korpus trianguliert folglich drei verschiedene Datenarten, die sich wechselseitig ergänzen und als Erklärungsressource dienen können. Mit der Erhebung des ethnographischen Zusatzmaterials sowie der Fokusgruppendiskussion ist keine Privilegierung der Sicht der Lehrpersonen für die Analyse der Daten verbunden. Vielmehr erweisen sich diese Daten als notwendige Ressource, um entsprechend des Konzepts der „ethnographischen Gesprächsanalyse“ (Deppermann 2013), dem hier gefolgt wird, die verschiedenen Wissensbestände des*der Analytiker*in und des*der Teilnehmer*in auszubalancieren.

Das methodische Vorgehen in der Auswertung der videographierten Interaktionsdaten aus dem Seminar erfolgt datensensitiv und qualitativ-sequenzanalytisch im Paradigma der multimodalen Interaktionsanalyse (z. B. Deppermann 2008, 2013, 2018) sowie gegenstandsangemessen auf schreibwissenschaftlich relevante Fragen zugeschnitten (Grieshammer 2017). Dazu sind die Daten inventarisiert und nach GAT2 (Selting und Auer et al. 2009) sowie multimodal (Mondada 2016) transkribiert worden. Das ethnographische Zusatzmaterial wurde inventarisiert und codiert; die Fokusgruppendiskussion wurde transkribiert und codiert; ausgewertet wurde beides mit den Mitteln der qualitativen Inhaltsanalyse unter Nutzung der Software MaxQDA (Rädiker und Kuckartz 2019). Das Korpus gestattet somit, neben der Bearbeitung des vordringlich interaktionstypologischen Interesses, vielfältige weitere Erkenntnismöglichkeiten.

\footnotetext{
${ }^{2}$ Das wird sichtbar in den Transkriptausschnitten in den Abb. 2-15.
} 


\section{Analysen und Diskussion}

In den folgenden Abschnitten werden Feedbackpraktiken beschrieben, die zeigen, wie im Auswertungsgespräch Feedback prozessiert wird, indem es auf die Konstitutionseigenschaften von Gesprächen, auf Sequenzialität und Interaktivität, reagiert. Gefolgt wird dem mikrostrukturellkonversationsanalytischen Praktikenbegriff, wonach Praktiken multimodale, kontextgebundene Konstituenten von Handlungen sind (Deppermann et al. 2016, S. 13); der Praktikenbegriff erlaubt zudem, Materialität, Medialität, Beteiligungsstrukturen, Routinisierung und Prozessualität zu integrieren (Deppermann et al. 2016), wie es notwendig ist, wenn konkreter (Sprach-)Gebrauch in leiblicher, räumlich ko-präsenter Interaktion analysiert wird. Feedbackpraktiken konstituieren in diesem Sinn die komplexe Handlung des Auswertens.

Es werden im Folgenden zwei Schwerpunkte gesetzt. Erstens geht es um die Handlungsaufgabe des Etablierens der Reflexionsebene und der (möglichen) Perspektiven auf den Text, eng verknüpft mit Aktivitäten zu Problematisierung einer Textstelle (siehe Abschn. 4.2.). Gezeigt wird die systematische Bearbeitung dieser Handlungsaufgabe in den Eröffnungen der Auswertungsgespräche. Darin wird am Auswertungsobjekt ein relevantes Phänomen für die Auswertung ausgewählt, benannt und als ein gemeinsames Objekt etabliert. Aufgezeigt wird, wie die Bedingungen für die Möglichkeit für Reflexion und Feedback geschaffen werden, um die Auswertungen produktiv werden zu lassen, da es in den zugrundeliegenden Daten nicht darum geht, bestimmte definierte Werkzeuge des Feedbacks anzuwenden, sondern vor allem das Auswertungsgespräch als das Reden über Texte mit Feedbackfunktion zu prozessieren und somit spezifische Erkenntnis zu ermöglichen.

Zweitens wird ein besonderes und zugleich zentrales Phänomen - der Umgang mit der Autorschaft eines Textes herausgearbeitet (siehe Abschn. 4.3.). Dieses Phänomen hat insofern gegenstandsbezogen Relevanz, da hierbei die Perspektive auf den Prozess und seine Akteur*innen in Konflikt mit der produktorientierten Sicht auf das Auswertungsobjekt, den Text, gerät. Der ambige Umgang mit Autorschaft ist Ausdruck der Hybridität des Interaktionstyps zwischen Lehre und Coaching.

Limitationen für den vorliegenden Artikel bestehen zum einen in der Notwendigkeit zur Auswahl. Die beiden Schwerpunkte sind Ausdruck einer notwendigen Wahl von Phänomenen, die erstens nach ihrer schreibwissenschaftlich-gegenstandsbezogenen Relevanz sowie nach ihrer Salienz getroffen wurde sowie zweitens nach ihrem Potenzial, die Schnittmenge zum Coachingprozess aufzuzeigen. Damit ist nicht gesagt, dass das alle vorfindlichen Feedbackpraktiken sind, vielmehr hält das umfangreiche Korpus weitaus mehr bereit; auch das Modell des Setting der Auswertungssituation, wie im folgenden Abschn. 4.1. dargestellt werden wird, offenbart weitere Anschlussmöglichkeiten.

\subsection{Die Auswertungssituation}

Im semestralen Verlauf eines Seminars ist Auswerten kein eigenständiger Themenschwerpunkt mit einem progressionsbezogen bestimmbaren Platz, sondern ein Arbeitsschwerpunkt, der gestellten Aufgaben folgt und mehrfach im Seminar etabliert wird. Es handelt sich um eine als Interaktionstyp beschreibbare, wiederkehrende, aufgabenbezogene Einheit, die sich durch hohe Stabilität in Bezug auf das Interaktionsensemble, die Verfahren und Praktiken auszeichnet. Das zeigt Abb. 1. Das aus den Analysen der Daten extrahierte Schema zeigt, aus welchen Elementen und Prozessen sich das stabile Setting des Auswertungsgesprächs zusammensetzt, wobei Produktions- und Rezeptionsspezifika sowie die materialen Eigenschaften des Auswertungsobjekts (i.e.S. Text oder Rede/Gespräch) und die jeweiligen Affordanzen gleichermaßen in den Blick genommen werden, ebenso die jeweiligen theoretischanalytischen Perspektiven (oberste Zeile) darauf. Die Komplexität des gesamten Settings lässt sich hier nicht erläutern, wird aber modellhaft in der Grafik sichtbar.

Im Folgenden sollen die beiden für die Analysen im vorliegenden Aufsatz relevanten Aspekte hervorgehoben werden. Das ist zum einen die Verbindung zwischen Auswertungsobjekt und Auswertungsgespräch sowie zum anderen die Beteiligungsstruktur, konkret der Bezug der Auswertungsperspektiven auf die Autorschaft eines Textes (gepunktete Linie in Abb. 1).

Die Verbindung zwischen Auswertungsobjekt und Auswertungsgespräch verweist auf vorgängige Schreibprozesse und ist zugleich wesentliches Element des Auswertungsgesprächs, in das die Schreibaufgabe integriert ist. Bei den Aufgaben handelt es sich um komplexe Aufgaben im Sinne Bachmann und Becker-Mrotzek (2010, S. 191), die sich als Projektionsfläche für Reflexion eignen und verschiedene Aktivitäten des Reflektierens anregen müssen (Schwarze und Rieder 2014, S. 453). Aus der Aufgabe erwächst ein Produkt, das ein Text oder eine Textform sein kann. Mit Bezug auf Pohl und Steinhoff (2010, S. 6) werden darunter Schreibanlässe in Bildungskontexten verstanden, die das Verfassen von Texten nach sich ziehen, nicht jedoch zwingend solchen, die einer Textsorte zugeordnet werden können; in diesem Sinn sind Textformen Lernformen, die spezifische, schreibdidaktisch funktionale epistemische Prozesse auslösen. Das Produkt muss aktiv im Auswertungsprozess zu einem Auswertungsobjekt zugeschnitten werden. Das Auswertungsobjekt ist konstitutiv für die Ko-Konstruktion von Veränderungsprozessen, da es einen materialen Be- 


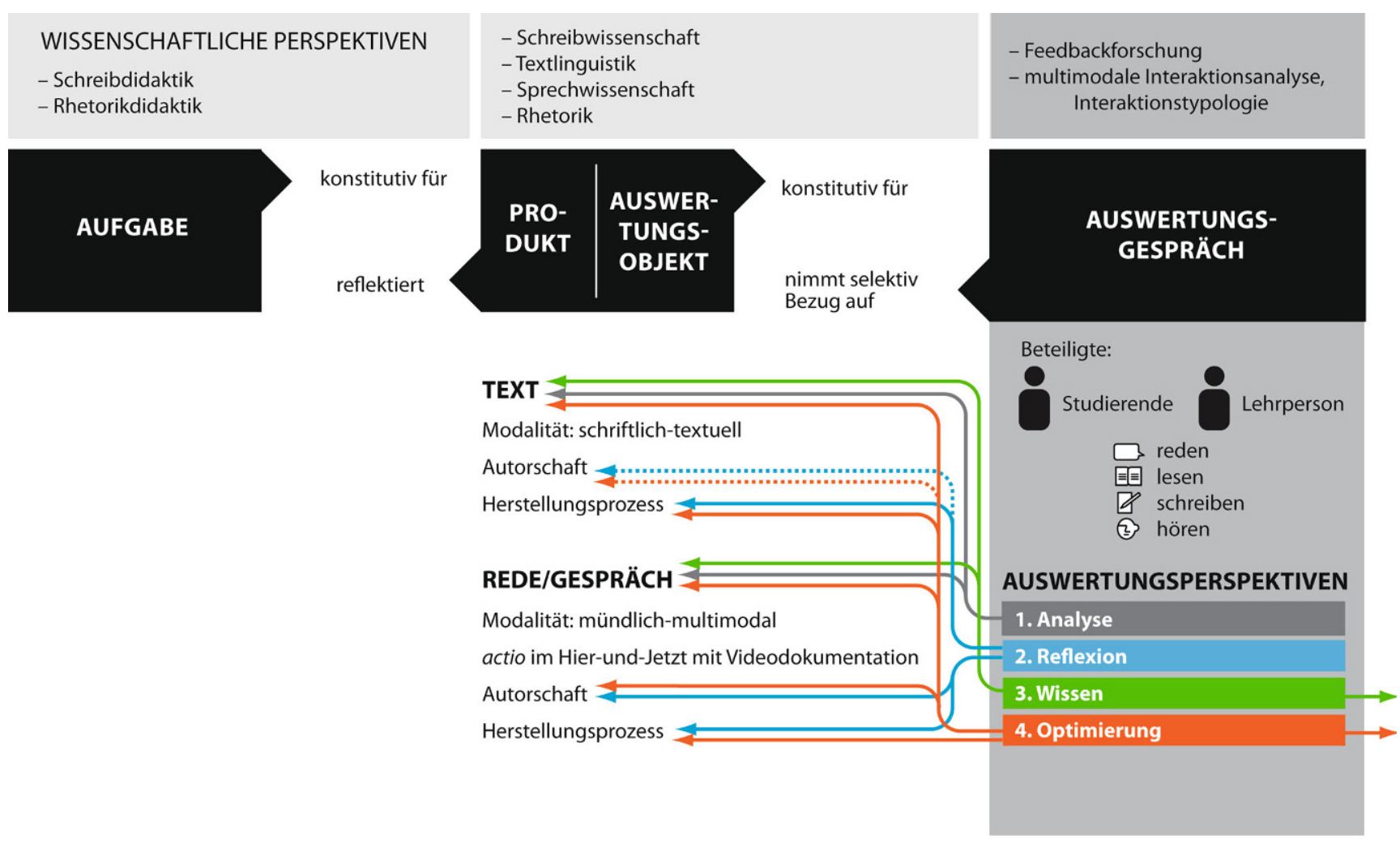

ZEIT

Abb. 1 Elemente und Prozesse der Auswertungssituation

zugs- bzw. Startpunkt darstellt und durch Autorschaft erfahrungsgebundene Reflexion ermöglicht.

Die interaktive Beteiligungsstruktur des Auswertungsgesprächs und die damit verbundenen möglichen Beteiligungsweisen können mit dem Konzept des „Interaktionsensembles" (Deppermann und Schmitt 2007, S. 20) gefasst werden. Die Beteiligungsweisen leiten sich aus dem Mehrpersonengespräch des Seminars (anders als z.B. das Zweiergespräch in der Schreibberatung oder im Einzelcoaching) ab sowie aus verschiedenen Asymmetrien. So lässt sich die Beteiligungsweise der Lehrperson als die einer „Fokusperson“ kennzeichnen, das ist „eine(n) Interaktionsbeteiligte(n), die/der aufgrund von Status, Funktion oder Rolle in bestimmten Kontexten kontinuierlicher Bezugspunkt von Monitoring-Aktivitäten anderer Beteiligter ist“ (Deppermann und Schmitt 2007, S. 35); mit dem Terminus Fokusperson werden die Wahrnehmungsverhältnisse und die Interaktionskonstellation betont. Darüber hinaus gehört die Integration von Objekten wie Texten in der Interaktion sowie die Feedbackstruktur als das Geben (Beteiligungsweise der Feedbackgebenden) und Nehmen (Beteiligungsweise der Referenzperson) von Feedback dazu. Die Interagierenden der Auswertungsgespräche sind folglich als eine community of practice (Lave und Wenger 1991) konzeptualisierbar.

\subsection{Die Eröffnung des Auswertungsgesprächs: Das Etablieren der Reflexionsebene}

Eröffnungen (und Beendigungen) von Gesprächen stellen sicher, dass ein Gespräch als singuläres Ereignis für die Interagierenden wahrnehmbar wird. Die Eröffnungen der Auswertungsgespräche können als „Situationseröffnungen“ bezeichnet werden, ein spezifischer Fall der ,,multimodalen Herstellung fokussierter Interaktion" (Mondada und Schmitt 2010, S. 8). In den Eröffnungen müssen das Interaktionsensemble, die Beteiligungsweisen und das Auswertungsobjekt konstituiert werden, daher präsentiert sich das Eröffnen als eine prospektiv koordinative Aktivität. Das Auswertungsobjekt eröffnet in seiner spezifischen medialen Verfasstheit - ausgeteilt als Blatt oder sichtbar an der Projektionsfläche - auch Erwartungsstrukturen insofern, dass auf die vom ihm ausgehenden Affordanzen reagiert werden muss.

Eröffnungen sind auch aus der Perspektive der Coachingprozessforschung bearbeitet worden (z. B. zu Identitäten und Beziehungsgestaltung im Erstgespräch vgl. Rettinger 2011). Vor allem aber können Aktivitäten in den Eröffnungen der Auswertungsgespräche mit der für das Coaching herausgearbeiteten kommunikativen Basisaktivität „Definieren der Situation“ in Verbindung gebracht wer- 


$\begin{array}{ll}0004 \text { LP } & \text { ihre HAUSaufgabe WAR es } \\ 0005 & \text { (.) die FRAge <<zitierend> WAS ist das zÜRcher } \\ & \text { TEXTanaLYseRAster; } \\ 0006 & \text { auf der basis der informationen- } \\ 0007 & \text { die sie dem (--) ahm (--) parLANdoartikel von SIEber } \\ & \text { entNEHmen KONNten- } \\ 0008 & \text { (.) KURZ zu beANTworten- } \\ 0009 & \text { also quasi eine definition dieses beGRIFfes (.) ZÜRcher } \\ & \text { TEXTanalYseRASter (.) zu LIEfern. }\end{array}$

Abb. 2 Aus: schr_1411 (00:00:03-00:00:23)

den (Graf 2015, S. 11). Darunter fasst Graf orientierende Handlungen zu Zweck und Gestaltung der Interaktion für die Beteiligten sowie verfahrenspraktische Aspekte wie beispielsweise Terminvereinbarungen. Ähnliches gilt für die Situationseröffnungen in den Auswertungsgesprächen, sie dienen der Orientierung und Einbettung der Interaktionsphase in das größere Ganze des Seminars, der Etablierung der Reflexionsebene sowie der Klärung verfahrenspraktischer Aspekte.

Auf der Basis der hier genutzten Daten kann die Spezifik der Situationseröffnungen über alle Auswertungsgespräche im Teilkorpus zum Schreiben hinweg sequenzanalytisch rekonstruiert werden. Dabei lassen sich vier Handlungsaufgaben rekonstruieren, die in komplexen Sequenzmustern prozessiert und systematisch in dieser Reihenfolge mesosequenziell abgearbeitet werden, wobei sich verschiedene Detaillierungsgrade zeigen:

1. Herstellen des Rückbezugs zur Schreibaufgabe und Reformulierung der Aufgabenstellung

2. Thematisierung der subjektiven Einschätzung des Erfolgs und des Prozesses

3. Transparenzherstellung hinsichtlich der auswertungsverfahrensorganisatorischen Bearbeitung durch eine auswertungsobjektsensible Verfahrensexplikation

4. Information über die Auswertungsschwerpunkte für das Auswertungsgespräch.

Diese Handlungsaufgaben bringen koordinierte Verfahrensweisen hervor, um die Eröffnung in die Kernsequenz des Auswertungsgesprächs hinein gut vorzubereiten und zugleich Reflexion und Feedback im Gespräch zu ermöglichen. Alle vier Handlungsaufgaben werden im Folgenden anhand von kollektionierten Transkriptausschnitten aus verschiedenen Interaktionsdokumenten illustriert, wobei auf die Gemeinsamkeiten, aber auch auf die Varianz in den Ausgestaltungen besonderes Augenmerk gerichtet wird.

\subsubsection{Schritt 1: Herstellen des Rückbezugs zur Schreibaufgabe und Reformulierung der Aufgabenstellung}

Mit dem Herstellen eines Bezugs zur Schreibaufgabe und der Reformulierung der Aufgabenstellung wird der Gegenstandsbezug etabliert. Die Orientierung ist retrospektiv, das Kommende wird mit der vorgängigen Arbeitseinheit verknüpft und so Kontinuität im Seminar hergestellt. Zugleich stellen alle Lehrpersonen in der Bearbeitung dieser Handlungsaufgabe die Fachlichkeit der Aufgabe her, indem auf die für den Wissensaufbau relevanten Texte, die Grundlage der Schreibaufgaben waren, erneut verwiesen wird. Formal enthalten die Rückbezüge eine auf die Reformulierung der Aufgabe hinführende Äußerung (z. B.: es ging ja darum, ihre HAUSaufgabe WAR es). Der Rückbezug kann sehr knapp gestaltet sein oder stärker ausgebaut wie im Transkriptausschnitt in Abb. 2, der die Benennung als Hausaufgabe enthält und die Aufgabenstellung zitiert; auf diese Weise ist der Lernkontext in der Gegenwart aktualisiert.

In schreibdidaktischer Perspektive handelt es sich um eine Lese- und Schreibaktivitäten verschränkende Aufgabe, die das Verfassen der Textsorte Definition fokussiert und einen fachlich relevanten Text $^{3}$ als Arbeitsgrundlage hat; so wird zugleich auch fachspezifische Wissensarbeit geleistet. Im Auswertungsgespräch wird nun in spezifischer Weise Anschlusskommunikation hergestellt und so einem reflexionsorientierten Ansatz gefolgt.

\subsubsection{Schritt 2: Thematisierung der subjektiven Einschätzung des Erfolgs und des Prozesses}

Mit Frage an die Studierenden nach der subjektiven Einschätzung des Erfolgs ihrer Aufgabenbewältigung wird die Ebene der Reflexion des eigenen Handelns für das Auswertungsgespräch erstmalig eingebracht. Auch dieser Schritt wird in jeder Eröffnung durchgeführt und ist variantenreich

\footnotetext{
${ }^{3}$ Dabei handelt es sich um den Text von Sieber (2008). Hinweisen möchte ich darauf, dass dieses Wissen nur durch die Erhebung des ethnographischen Zusatzmaterials für die Analysen verfügbar ist.
} 
027 LP (.) so wie ist es ihnen geLUNgen-

Abb. 3 Aus: schr_0506 (00:01:43-00:01:44)

gestaltet. Die gestellte Frage rekurriert auf eine Unterscheidung, die von Dengscherz (2019, S. 166) anhand mehrsprachiger Schreibprozesse erneut fruchtbar gemacht wurde und sich gut auf die vorliegenden Daten übertragen lässt. Dengscherz (2019) unterscheidet zwischen Anforderungen und Herausforderung im Schreibprozess; während Anforderungen sich aus der Schreibsituation und Textsorte bzw. Aufgabe ergeben, beziehen sich Herausforderungen auf die subjektive Einschätzung der Bewältigbarkeit der Anforderungen und eine Einschätzung der eigenen Ressourcen. Die Thematisierung der subjektiven Einschätzung verweist also auf die wahrgenommenen individuellen Herausforderungen und etabliert den Blick auf die individuelle Handlungsfähigkeit.

Zum einen ist diese Aktivität als nicht eingebettete, prospektive Ankündigung auf die im Gespräch nachfolgende Reflexion und Auswertung wie im obigen Beispiel in Abb. 3 gestaltet. Diese Frage wird gesprächsorganisatorisch nicht als Frage behandelt, denn weder wird sequenziell von der Lehrperson ein Sprecherwechsel angeboten, noch melden sich Studierende zu Wort. Vielmehr wird von der Lehrperson das Rederecht weiterhin beansprucht und mit der nächsten Handlungsaufgabe, die Auswertungsschwerpunkte $\mathrm{zu}$ etablieren, weitergemacht.

Zum anderen kann die Bearbeitung dieses Handlungsschrittes breiteren Raum einnehmen und vor allem interaktiv bearbeitet werden, wenn die konditionelle Relevanz der Frage bearbeitet wird wie im Folgenden anhand von drei Ausschnitten (Abb. 4, 5 und 6) aus einem einzigen Auswertungsgespräch sichtbar wird. Da wird die Frage nach der subjektiven Einschätzung des Arbeitsprozesses und seines Erfolgs zweimal gestellt: Zuerst als prospektiver Verweis (Abb. 4); eingebettet in den Verweis auf die Schreibaufgabe (in Abb. 4 Z. 23: Illokutionsstrukturanalyse) wird in Zeile 26-27 die subjektive Einschätzung erfragt, allerdings vorerst ohne einen Sprecherwechsel zu ermöglichen.

Wenige Äußerungen später (Abb. 5) schließt die Lehrperson selbstkongruent an die zuvor in den Zeilen 26-27 projizierte Frage an und reformuliert sie unter Spezifikati- on dessen, was berichtet werden soll, nämlich ,eindrücke und erfahrungen“ (Z. 61). Die Lehrperson beendet ihre ÄuBerung mit deutlichen Turnübergabesignalen, sodass sich der*die erste Student*in (S1) für einen Beitrag vorschlagen kann. Da diese Frage als Frage behandelt wird, ist der interaktive Raum, um zu antworten, eröffnet. In Zeile 64 beginnt S1, seine*ihre subjektive Einschätzung darzulegen und über eigene Erfahrungen zu berichten.

S1 gibt dem Bericht i.S. eines story preface die Rahmung, dass es eine ehrliche Einschätzung sei. Die Ankündigung: ,also wenn i_jetz ehrlich sein soll“ (Z. 64) sichert das Rederecht und projiziert eine potenziell problematische Fortsetzung, die in einem weiten Vorgriff durch die abschließende Evaluation in Zeile 82 als geschlossen gelten kann: ,und (.) $i$ hab probiert aber (.) bin gescheitert“. Dazwischen berichtet S1 von seinen*ihren handlungsbezogenen Versuchen, die Aufgabe so zu erfüllen, wie sie als Beispiel im Unterrichtsmaterial zu sehen war, berichtet von mehreren Versuchen, zeigt Ausdauer auf und benennt schließlich Verstehensschwierigkeiten der Aufgabenstellung.

Die von S1 aufgezeigten Verstehens- und Wissensdefizite bis hin zur unrichtigen Benennung des Fachterminus Illokution (Z. 78-79: illokation) werden von der Lehrperson (ab Z. 83 ff.) lokal nicht bearbeitet. Dadurch wird die etablierte Reflexionsebene zunächst aufrechterhalten und nicht verlassen, um beispielsweise das terminologische Defizit zu bearbeiten. Der Beitrag von S1 dient der Lehrperson jedoch als ein take off, um in der Handlungs- und Sequenzlogik einen Schritt zurück zu gehen und ausführlich die Arbeitsaufgabe der Illokutionsstrukturanalyse zu begründen sowie deren linguistisches und logisches Fundament aufzuzeigen (in Abb. 6). Darin konzediert die Lehrperson mehrfach, dass die Aufgabe schwer gewesen sei. Diese fachliche Erklärung der Aufgabe schließt die Lehrperson ab, wie im Transkriptausschnitt in Abb. 6 zu sehen ist, indem sie das Fazit von S1 in Zeile 82 - probiert gehabt zu haben, aber zu scheitern aufgreift und ihr eigenes Handeln verdeutlicht (Abb. 6).

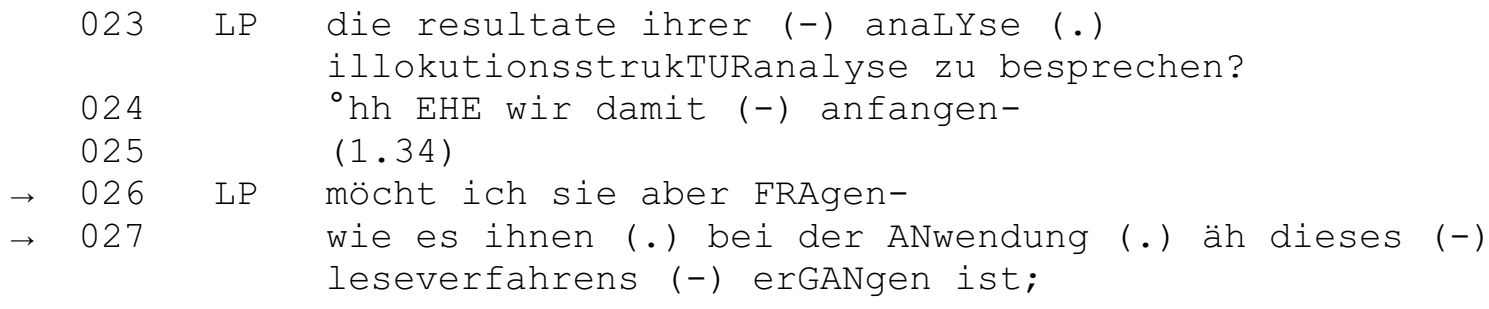

Abb. 4 Aus: schr_2510 (00:00:43-00:01:09) 


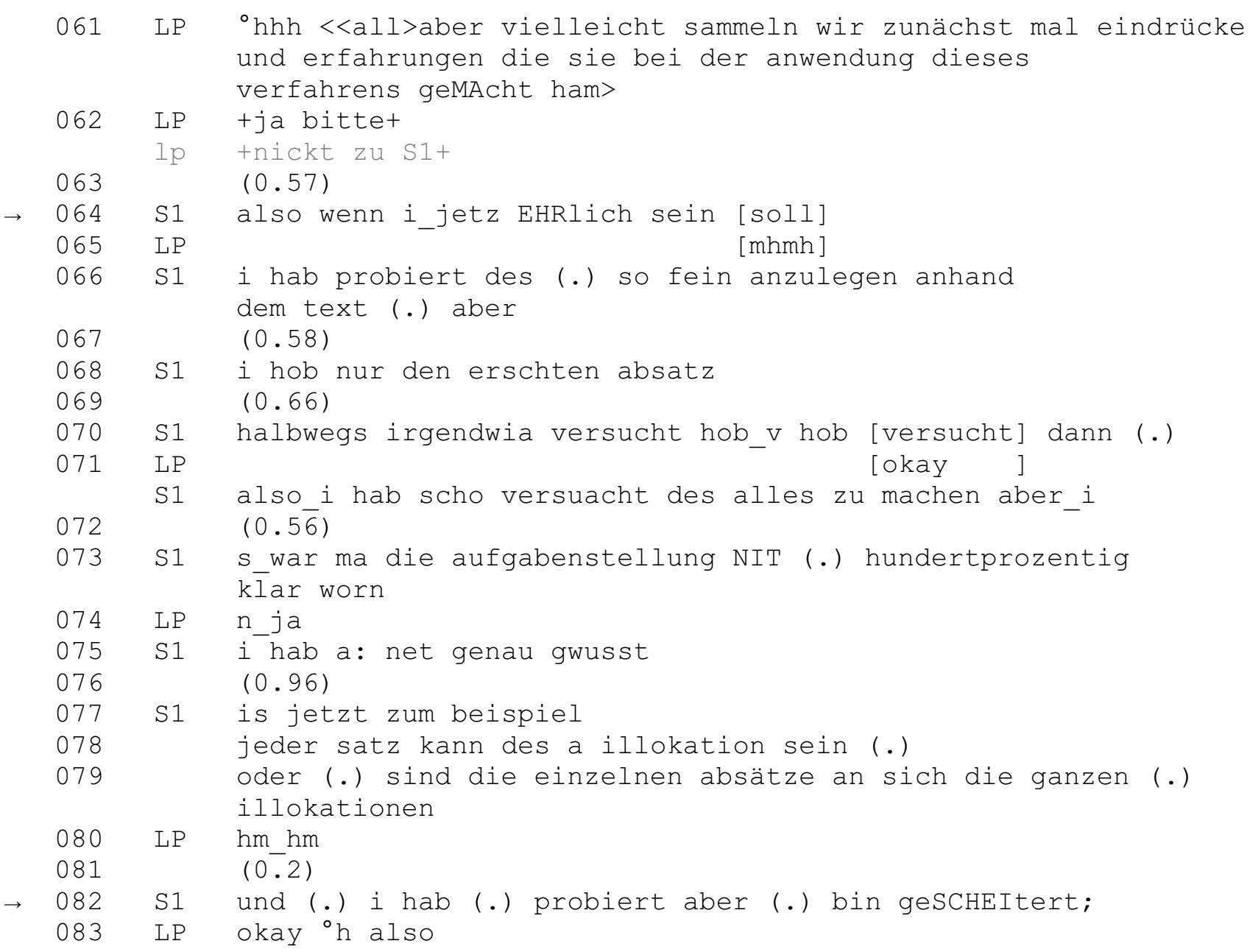

Abb. 5 Aus: schr_2510 (00:01:44-00:02:23)

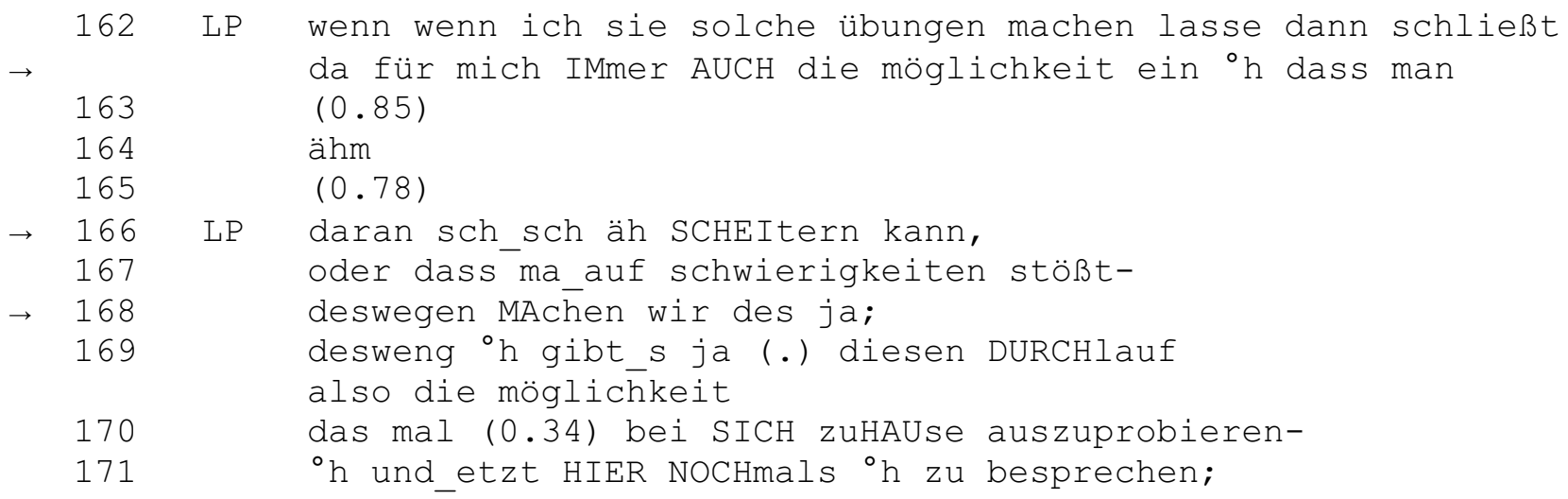

Abb. 6 Aus: schr_2510 (00:04:38-00:04:53)

Deutlich wird vor allem an dem, was die Lehrperson nicht tut - erklären oder korrigieren - ihre manifeste Orientierung an der Herstellung von Handlungsfähigkeit der Studierenden in Bezug auf das Schreiben. Sie sichert die Herstellung der Bedingungen der Möglichkeit zu individu- ellen Veränderungsprozessen. Das Scheitern wird produktiv gewendet und als Rechtfertigung für ein Auswertungsgespräch dargestellt. Funktional eröffnet sich sequenziell verortet hier eine Möglichkeit für die Lehrperson, Problembewusstsein für Schreibschwierigkeiten herzustellen. Das 
ist etwas, das für Schreibcoaching oder Schreibberatung konstitutiv für den Eintritt in die Interaktion ist. In diesen Gesprächen muss es jedoch erst hergestellt und etabliert werden.

Dadurch sind die beiden Handlungsaufgaben ,Reformulierung der Arbeitsaufgabe " und ,Reflexion des eigenen Erfolgs ' beendet und die Lehrperson kann mit der Prozessierung der Eröffnung fortfahren.

\subsubsection{Schritt 3: Transparenzherstellung hinsichtlich der auswertungsverfahrensorganisatorischen Bearbeitung}

Die Handlungsaufgabe des Herstellens von Transparenz in der auswertungsverfahrensorganisatorischen Bearbeitung umfasst das Offenlegen des Transformationsprozesses des Produkts als Ergebnis der Aufgabenstellung hin zum Auswertungsobjekt und ist, da sie in den Daten zum Schreiben (anders als in den Daten zum Rhetorikcoaching) nicht interaktiv bearbeitet wird, eine Aufgabe der Lehrperson. $\mathrm{Zu}$ dieser Aufgabe gehört auch die auswertungsobjektspezifische, deontisch konnotierte Verfahrensexplikation wie Hinweise auf die Geltung von Feedbackregeln, mithin eine kommunikationsethische Grundlegung sowie Fragen zum Umgang mit der Kategorie der Autorschaft. Das erfolgt zumeist als Verweis auf die Anonymisierung der Texte (ausführlicher in Abschn. 4.3.).

Das Herstellen von Transparenz in der auswertungsverfahrensorganisatorischen Bearbeitung wird oft knapp und ohne Begründung realisiert und ist von körperlich-räumlicher Aktivität der Lehrperson wie z. B. dem Austeilen der Auswertungsobjekte begleitet. In Abb. 7 wird von der Lehrperson retrospektiv auf ihre Auswahl von Texten aus den Lösungen verwiesen; in Abb. 8 erfolgt ein Verweis auf die eigenen, durchgeführten Bearbeitungsschritte (Z. 45-47). Prospektiv wird knapp instruiert, indem die Lehrperson be- nennt, wie damit im Folgenden verfahren werden soll (anschauen und besprechen, anschauen).

Es sind zunächst sehr allgemeine Instruktionen, die erst bei der Bearbeitung der nächsten Aufgabe - Zuschnitt des Auswertungsgegenstands und der Auswertungsschwerpunkte für die Weiterarbeit - konkretisiert werden.

\subsubsection{Schritt 4: Information über die Auswertungsschwerpunkte für das Auswertungsgespräch}

Dieser Handlungsschritt erweitert auch die Handlungsaufgabe ,Reformulieren der Schreibaufgabe` um präzise und begründete Instruktionen darüber, was nun mit dem Auswertungsobjekt getan werden soll. Das mündet organisatorisch häufig in eine Arbeitsphase am Blatt allein oder in Kleingruppen, gefolgt von plenaren Gesprächen.

Das veranschaulicht der Transkriptausschnitt in Abb. 9, worin die Lehrperson die Auswertungsobjekte, die analyseleitenden Fragen sowie Auswertungsaufträge ableitet. Das ist zunächst, in den vier zur Auswertung ausgewählten Texten alle Nomen zu unterstreichen, ,die da geWÄHLT worden sind UM den beGRIFF ZÜricher TEXTanaLYseRASter $h^{\circ}$ zu defiNIEren“" (Z. 67-68).

Im nachfolgenden Beispiel (Abb. 10) hat die Lehrperson im lokalen Vorlauf der Textstelle die Merkmale der Wissenschaftssprache, wie sie die begleitende Lektüre zur Schreibaufgabe angibt, ausführlich reformuliert. Das mündet in die Instruktion in Zeile 55-56: „dass sie das einmal einfach verGLEICHen und sich das ANstreichen und danach REDen wir daDRÜber" als Aufgabe für eine längere Arbeitsphase am Blatt. Die genannten Merkmale der Wissenschaftssprache werden in der nachfolgenden Kernsequenz des Auswertens zu analyseleitenden Grundsätzen, andere werden nicht aufgemacht.

$\begin{array}{ll}0010 \text { LP } & (--) \text { ich HAB nun von IHren (--) LÖsungsvorSCHLÄgen- } \\ & \text { die sie ins OLAT gestellt haben VIER AUSgewählt- } \\ & \text { die wir jetzt ANschauen und beSPREchen wollen, } \\ 0011 & (1.3)\end{array}$

Abb. 7 Aus: schr_1411 (00:00:24-00:00:34)

$\begin{array}{lll}045 & \text { LP } & (--) \text { jetzt habe ich da aus ähm DREI verschiedenen } \\ 046 & & (0.8) \\ 047 & \text { LP } & \text { LÖsungen von IHNen einfach nur einzelne sätze heRAUsgenommen } \\ & \text { und gegenübergestellt, (-) } \\ 048 & & \text { den ersten text also den SUBjektiven, (-) } \\ 049 & \text { und den zweiten also die UMformulierungen; } \\ 050 & (-) \text { schauen sie sich mal AN, }\end{array}$

Abb. 8 Aus: schr_0506_1 (00:02:29-00:02:46) 


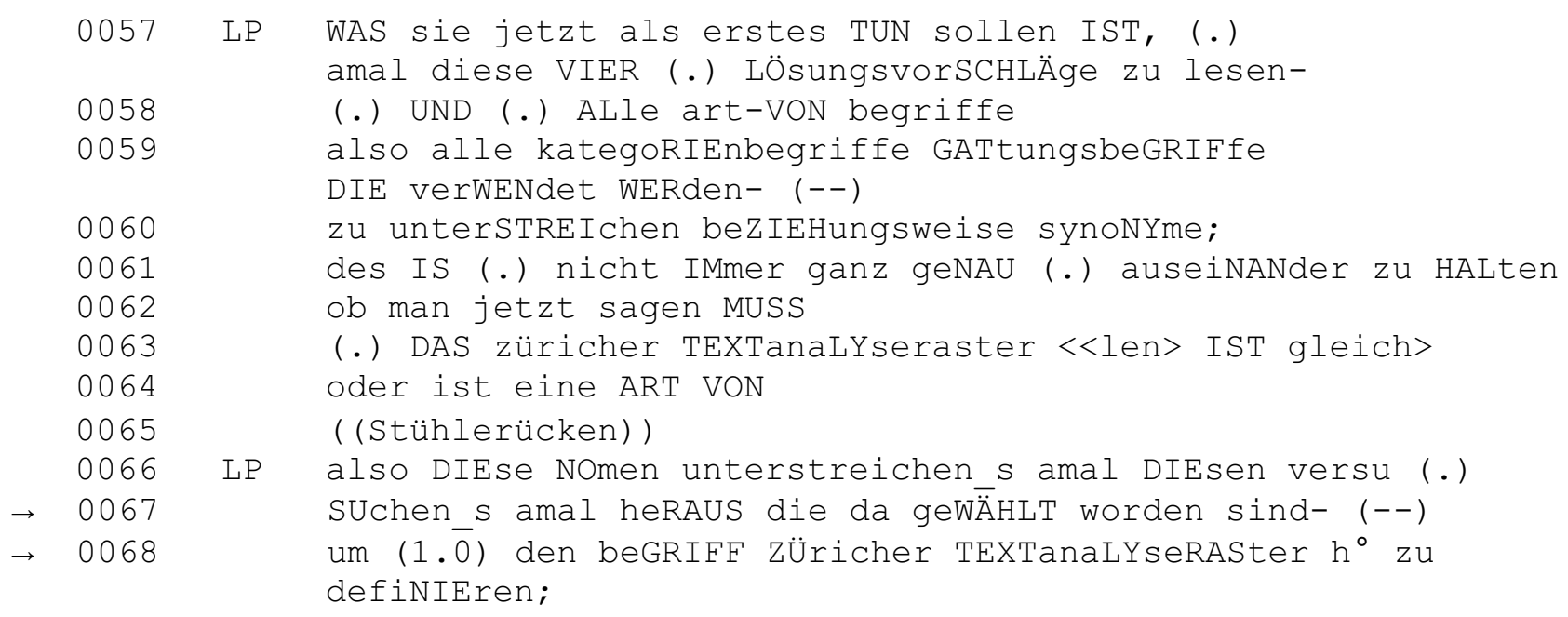

Abb. 9 Aus: schr_1411 (00:02:02-00:02:37)

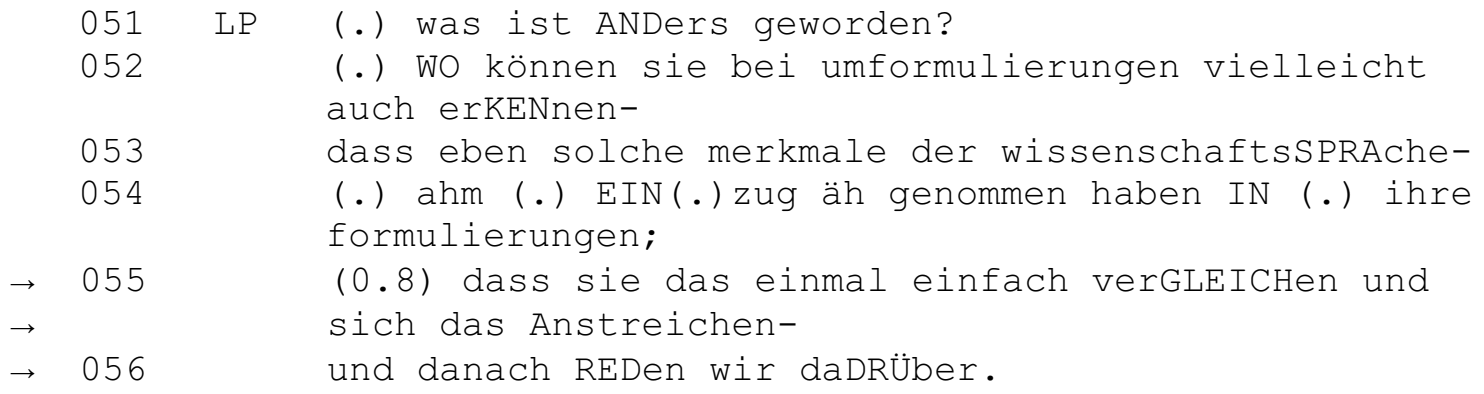

Abb. 10 Aus: schr_0506_1 (00:02:46-00:03:04)

Der typische Abschluss der letzten Phase der Eröffnungen ist eine Instruktion, die zugleich das opening der nachfolgenden Kernphase des Auswertens darstellt. Damit ist die Eröffnung beendet, worin die zukünftige Arbeit strukturiert und Wissensressourcen aktualisiert wurden. Auf diese Weise wird in das gemeinsame Arbeiten dieser etablierten community of practice (Lave und Wenger 1991) als einer lokal relevanten Lern- und Arbeitsgemeinschaft geführt.

De Daten zeigen, dass die Eröffnungen systematisch prozessiert werden, das diskursive Muster lässt sich qualitativ über alle Auswertungsgespräche hinweg ermitteln. Es ist eine Phase hoher Aktivität, v. a. von Steuerungsaktivitäten der Lehrperson, die hier vor allem als Moderator*in gefordert ist. Das ändert sich in den nachfolgenden Phasen, dort zeigt sich die Beteiligungsweise der Lehrperson stärker als die eines*einer Expert*in für Wahrnehmung und Beobachtung oder auch für Fachlichkeit.

Funktional wird das Auswertungsgespräch eingebettet und ein Rahmen geschaffen, der durch die spezifische Kopplung von analytischen Fragen an bzw. bezogen auf konkrete Reflexionsprobleme die spezifische Prozessierung von Feedback erst ermöglicht und daher als Phase in seiner Relevanz nicht zu unterschätzen ist.

\subsection{Der Umgang mit der Autorschaft eines Textes}

In diesem Abschnitt soll der Fokus auf die rekonstruierten Feedbackpraktiken im Umgang mit der Autorschaft eines Auswertungsobjekts gelegt werden. Das ist in zweierlei Hinsicht für das Schreibcoaching relevant: Erstens geht es um das schreibwissenschaftlich zentrale Konzept der Autorschaft eines Textes. Dies ist auch der Ansatzpunkt für Veränderung als einer Veränderung des konkreten Handelns einer Person, wie die prozessorientierte Schreibdidaktik betont (z. B. Ruhmann und Kruse 2014). Zweitens bedingt die interaktionsanalytische Perspektive auf das Interaktionsensemble und die Herstellung von Feedback die Relevanz des Fokus, da für die Studierenden zwei verschiedene Beteiligungsweisen vorgehalten werden, die der Referenzperson als derjenigen, die das Feedback erhält und die der Feedbackgebenden. Die Position der Referenzperson rekurriert daher auf die Kategorie der Autorschaft des Auswertungsobjektes Text. Im Folgenden sollen anhand von typischen Ausschnitten aus den Gesprächen die spezifischen Feedbackpraktiken, die mit der Autorschaft verknüpft sind, demonstriert werden; abschließend wird ihre Funktionalität herausgearbeitet: 
1. Unkenntlichmachen des Autors/der Autorin.

2. Aufdecken von Autorschaft im lokalen Kontext.

3. Elizitieren von hypothetischen Autorschaftsentscheidungen innerhalb von Bearbeitungsszenarien.

\subsubsection{Unkenntlichmachen des Autors/der Autorin}

Wie im Abschn. 4.2. zu den Situationseröffnungen bereits erwähnt, wird der Umgang mit der Autorschaft innerhalb der Aktivitäten des Herstellens von Transparenz in der auswertungsverfahrensorganisatorischen Bearbeitung in den Eröffnungen relevant gesetzt, zumeist als kurzer Verweis auf die Anonymisierung (Z. 4 in Abb. 11).

Für die Interaktion hat die Anonymisierung der Texte zur Folge, dass die Referenzperson ,unkenntlich“ und daher nicht direkt adressierbar ist. Sie hat somit keine aktiven Eingriffsmöglichkeiten im Gespräch und kann (zunächst) weder etwas nachfragen oder um Präzisierungen bitten. Der Schritt der Anonymisierung des Auswertungsobjekts hält also verschiedene Handlungsrestriktionen für die anwesenden Studierenden bereit, die von Lehrpersonen erklärt bzw. detailliert dargestellt werden, wie das Transkript in Abb. 12 zeigt.
Abschließend (Z. 20 in Abb. 12) werden von der Lehrperson (unter Verweis auf die im Seminar durchgängig gültige Regel) nachdrücklich die Texte selbst in den Mittelpunkt gesetzt und nicht ihre Autor*innen. Davor werden die Autor*innen instruiert, wie sie sich im Gespräch im Falle von Kritik zu verhalten haben. Für die Studierenden ist dieser Umgang mit der Autorschaft mit Geboten für den öffentlichen Umgang verknüpft (nicht outen, nicht rechtfertigen, nicht erklären, nicht sich selbst loben, sich heimlich freuen dürfen) und hat einen hohen Verpflichtungsgrad. Diese Verfahrensexplikation findet sich an vielen Stellen im Korpus und wird zwischen den Lehrpersonen unterschiedlich ausformuliert, jedoch von den einzelnen Lehrpersonen selbstkongruent nahezu wort- und formulierungsgleich hergestellt. Es handelt sich somit um eine Routineformel für lokal fundierte Feedbackregeln.

In funktionaler Sicht wird dabei jedoch eine Perspektive ausgeklammert, die in der besonderen Begegnung von Lesenden und Schreibenden liegt, worin Autor*innen Auskunft geben können und erfahren, wie der Text auBerhalb der eigenen Schreibsituation aufgenommen wird (z.B. Brown 2010, S. 72, Grieshammer 2018, S. 233). So ist es für die Studierenden hier nicht nur nicht absehbar (und folglich in Lernperspektive auch nicht konstruktiv planbar), ob der eigene Text zur Auswertung ausgewählt

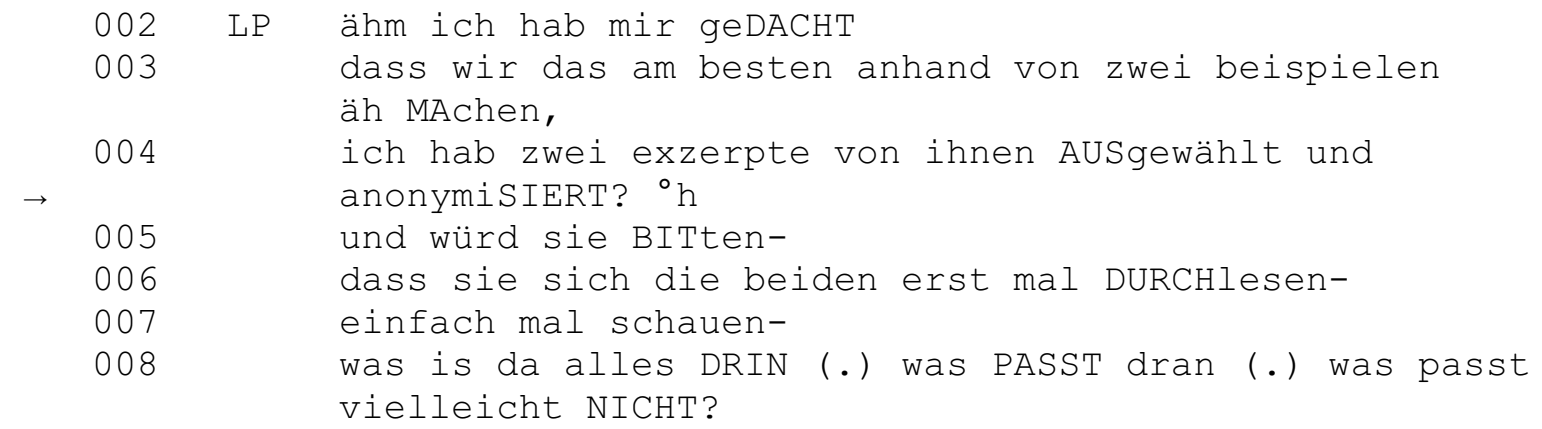

Abb. 11 Aus: schr_2911 (00:00:07-00:00:23)

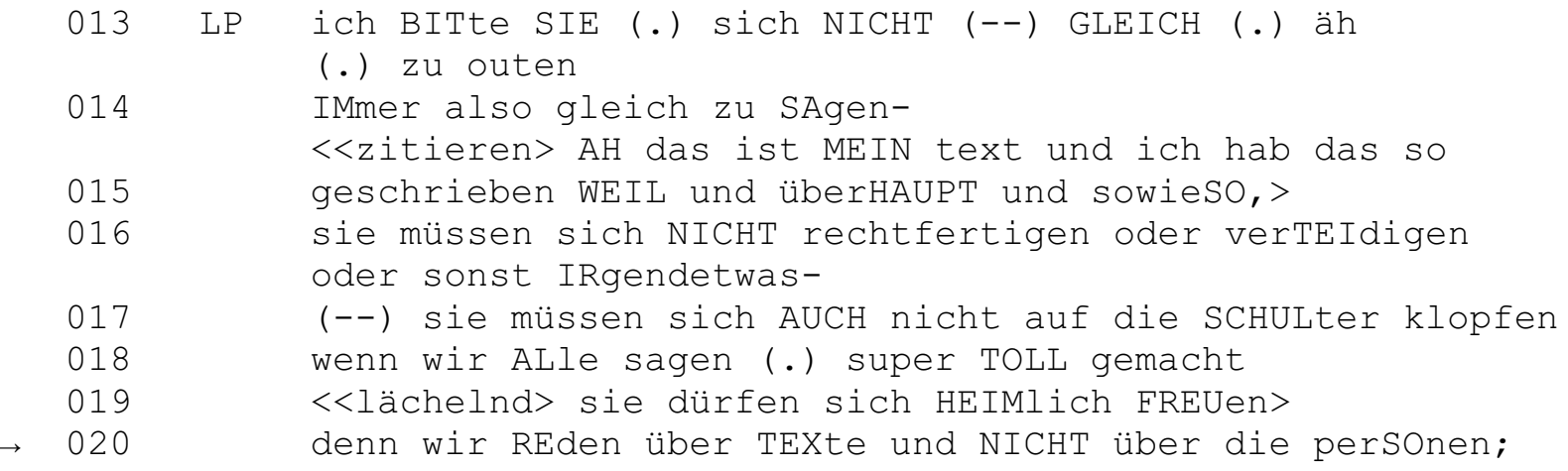

Abb. 12 Aus: schr_1411 (00:00:34-00:00:58) 
wird, sondern im Falle der Auswahl außerdem mit jenen spezifischen Restriktionen verbunden ist. Zugleich wird auf der Ebene des Sachverhaltsbezugs ein fachlicher Schwerpunkt gesetzt. In produktorientierter Perspektive, die den Fokus auf Texte und Textqualität richtet und eher Reflexionsaktivitäten als konkretes Feedback nahelegt, wird aber die prozessorientierte Sicht, die gerade in der Relevanzsetzung des Autors/der Autorin zum Vorschein kommt und ermöglicht, individuelle Handlungsfähigkeit zu erlangen bzw. zu verändern, hier anders integriert. Dies steht zuerst im Einklang mit textlinguistischen Konzepten als einer Disziplin ohne Schreiber*innen, steht aber im Widerspruch zu einem der Qualifikationsziele solcher Seminare, eben genau den anwendungsbezogenen Anteil zu stärken und den Kompetenzerwerb zu fördern.

Dass und auf welche Weise die Unkenntlichmachung des Autors/der Autorin von den Studierenden variantenreich unterlaufen wird, wird in den folgenden beiden $\mathrm{Ab}$ schnitten gezeigt.

\subsubsection{Aufdecken von Autorschaft im lokalen Kontext}

In den Daten finden sich systematisch kommunikative Praktiken, die sich zwar einerseits an das Gebot des sich nicht als Autor*in des besprochenen Textes zu erkennen zu geben halten, andererseits aber doch eigene Autorschaft lokal relevant setzen. Das geschieht zumeist ohne erhöhten kommunikativen Aufwand und funktional verschieden. Solche Feedbackpraktiken sollen an zwei typischen Beispielen gezeigt werden. Im ersten werden zwei anonymisierte Exzerpte eines fachwissenschaftlichen Artikels besprochen, vorbereitend werden die Textsortennormen des Exzerpts fokussiert. Im lokalen Vorlauf zum Transkriptausschnitt in Abb. 13 hat die Lehrperson der Gruppe Zeit gegeben, die beiden anonymisierten Exzerpte zu lesen. Die Lehrperson eröffnet die plenare Besprechungsrunde, indem sie entscheidet, mit welchem Text begonnen wird und fragt nach Evidenzen am Text, um Textreferenz herzustellen: „'hh was fällt ${ }^{\circ} \mathrm{h}$ uns denn da alles auf". S4 meldet sich gestisch zu Wort und bekommt es erteilt.

S4 äußert zunächst fragekonform etwas, was ihm*ihr aufgefallen ist: $d a$, also im ausgewerteten Text, sind keine Seitenangaben. Anschließend begründet S4 diese bemerkte Auffälligkeit mittels eines gestischen Verweises auf den eigenen Text, der auch vor ihr liegt (hier, Z. 27) und stellt Referenz zum Text her; er*sie bekräftigt die eigene Autorschaft daran und nennt das erhaltene Feedback, nämlich die Seitenzahlen noch dazuschreiben zu sollen (Z. 30). Hier wird der Verweis auf die eigene Autorschaft dazu genutzt, die eigene Beobachtung zu untermauern, da der Verweis unter Berufung auf die epistemische Autorität der Lehrperson als Begründung für die Richtigkeit der von $\mathrm{S} 4$ genannten Auffälligkeit fungiert. Von der Lehrperson wird nur die Wichtigkeit der Seitenzahlen bekräftigt, der Verweis von S4 auf sich selbst als Autor*in wird nicht explizit ratifiziert. Auf diese Weise hält die Lehrperson die Orientierung auf die beiden anonymisierten Texte.

Im nächsten Beispiel (Abb. 14) wird der Verweis auf die Autorschaft durch die Lehrperson selbst nahegelegt, indem nach dem Vorkommen eines Phänomens - formale Gestaltung von Verfasserreferenz in Texten - gefragt wird. Thematisch handelt es sich mit der Verfasserreferenz um eine schreibdidaktisch und textlinguistisch wesentliche Kategorie, die hohe Relevanz als Erwerbsgegenstand hat (z. B. Steinhoff 2007, S. 165).

Von der Lehrperson wird das textuelle Phänomen des sprachlichen Ausdrucks der Referenz auf sich selbst als auf den*die Autor*in des Textes diskutiert und dessen Vorkommen erfragt (Z. 12-13). S2 äußert in Zeile 15, es schon gesehen zu haben und markiert im Anschluss Nicht-Wissen über die Angemessenheit dieser sprachlichen Gestaltungsweise. Die Lehrperson bearbeitet sequenziell nicht das Wissensproblem der Angemessenheit, sondern verfolgt ihren eigenen, in den Zeilen 12-13 eröffneten Handlungsstrang und erfragt bei S2 ihm*ihr bekannte Evidenzen für eine solche Realisierung. Die dadurch hergestellte Textreferenz gilt jedoch nicht für das Auswertungsobjekt, sondern ruft

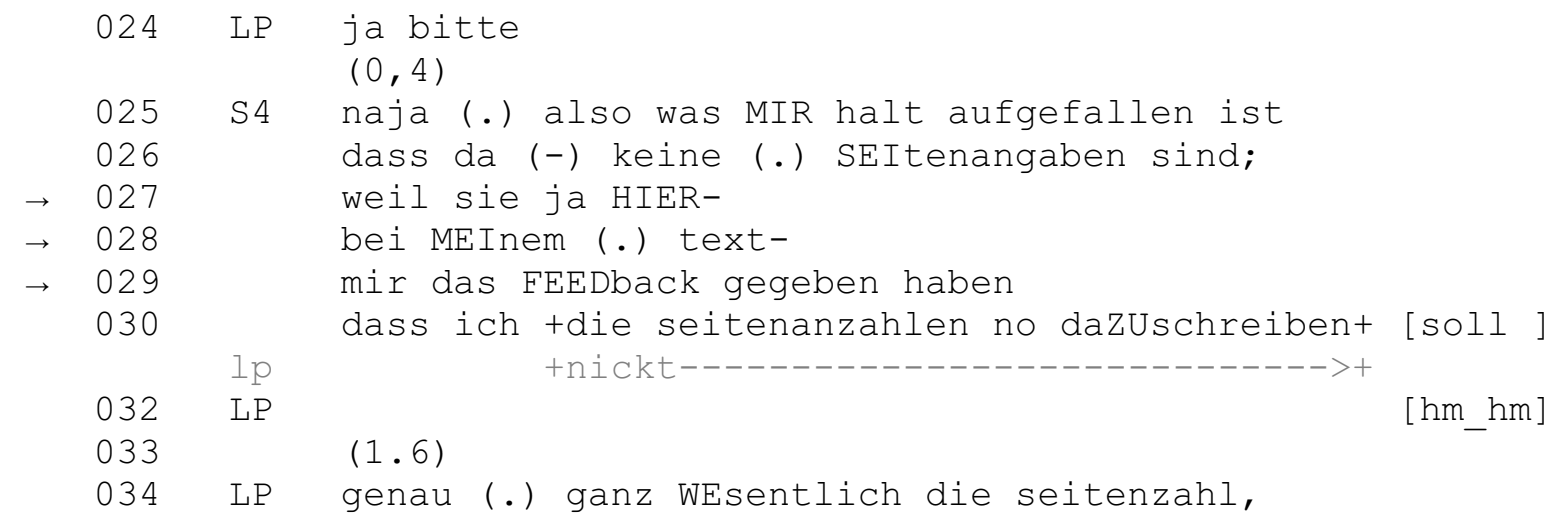

Abb. 13 Aus: schr_2911 (00:06:24-00:06:33) 


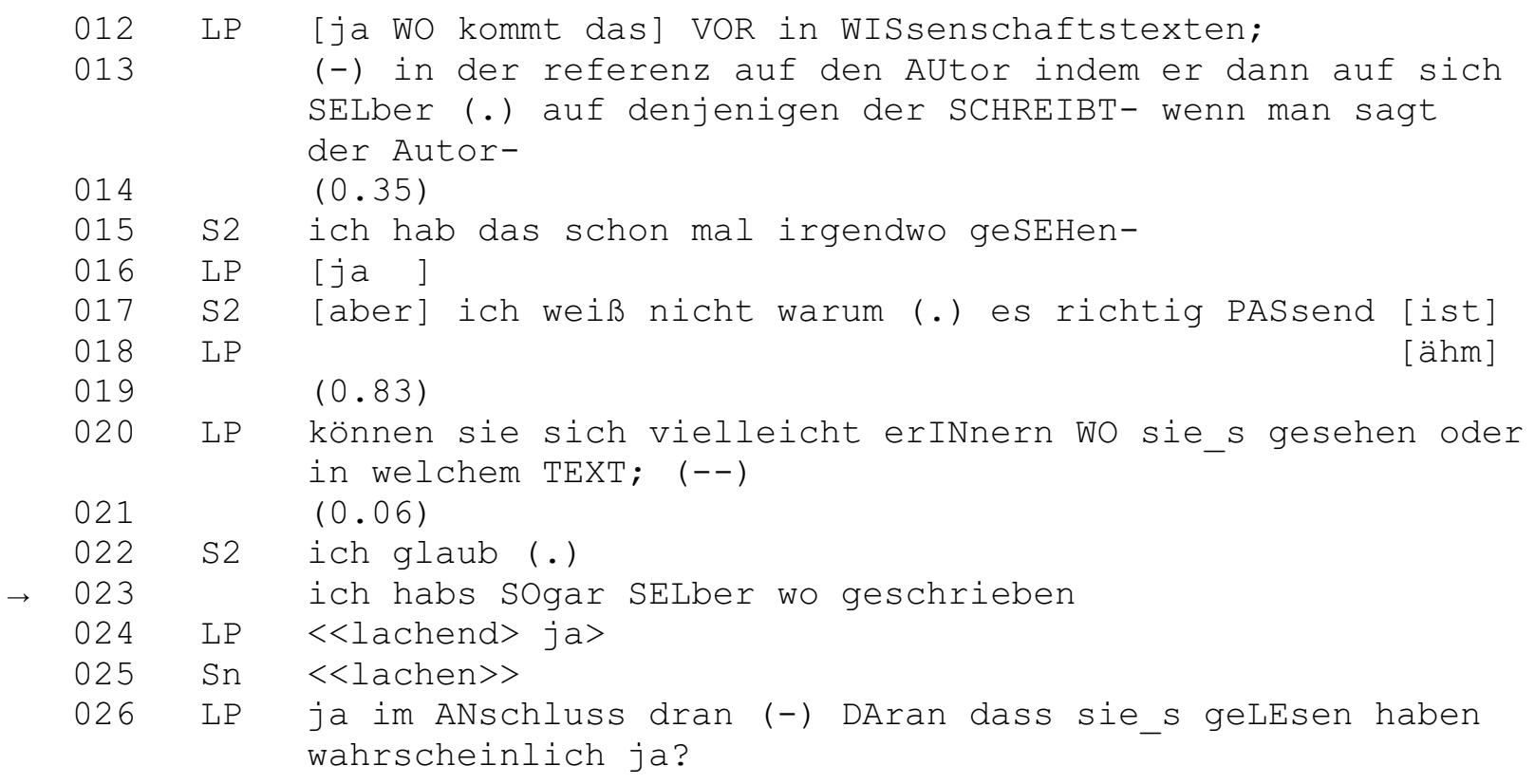

Abb. 14 Aus: schr_0506 (00:00:33-00:00:58)

generelle Leseerfahrung ab. Daraufhin (Z. 23) nennt S2 sich selbst eine*n Verfasser*in solcher Referenzen bzw. verweist auf einen eigenen Text (,SELber wo geschrieben“), der jedoch nicht der im Auswertungsgespräch besprochene Text ist. Damit positioniert sich S2 grundsätzlich als Autor*in von Texten. Das darauffolgende Lachen (Z. 25) auch der Lehrperson und der Studierenden greift die Differenz zwischen der Frage ,aber ich weiß nicht warum (.) es richtig PASsend ist" (Z. 17) und dem empirischen Vorkommnis im eigenen Text auf (Z. 23). Die eigentliche Frage nach der Regelbegründung wird weiter nicht bearbeitet. Stattdessen liefert die Lehrperson eine Vermutung der Vorgeschichte des Vorkommnisses (Z. 26) und beantwortet selbst die eingangs (im Transkriptausschnitt) von ihr gestellte Frage damit, dass dies in älteren Wissenschaftstexten der Fall sei. Dem folgt eine längere referierende Sequenz über den Wandel der Wissenschaftssprache; die Bearbeitung der Autorschaft ist beendet.

Beide Ausschnitte (Abb. 13 und 14) zeigen, dass und wie die Studierenden die lokalen Angebote, das eigene Handeln mit den Reflexions- und Feedbackaktivitäten anhand der Auswertungsobjekte abgleichen zu können, aktiv als Lernressource nutzen.

\subsubsection{Elizitieren hypothetischer Autorschaft}

Eine weitere Feedbackpraktik zum Umgang mit Autorschaft umfasst solche Bearbeitungsszenarien am Auswertungsobjekt, die hypothetische Autorschaft elizitieren. Dafür ist eine stellvertretende Perspektivenübernahme notwendig, die von den Studierenden leicht vollzogen werden kann, da die Schreibaufgabe von allen bearbeitet worden ist und daher auf der Basis der eigenen Aufgabenerfüllung (i.S. Wie haben Sie das gemacht?) geantwortet werden kann. Diese hypothetischen Überlegungen erfordern Verfremdung als Basis, diese Verfremdung wird durch die Anonymisierung der Texte geleistet.

Im Transkriptausschnitt in Abb. 15 ist die Frage der Lehrperson nach der Identifikation eigener Gedanken im Auswertungsobjekt (Z. 59, es geht hier um die Textsortenmerkmale des Exzerpts, im speziellen um die Unterscheidung des eigenen und des fremden Wissens) das Mittel, um Textreferenz herzustellen. S7 arbeitet im Beitrag ( $a b$ Z. 60) systematisch die gestellte Frage ab, indem er*sie zuerst aus dem Auswertungsobjekt vorliest, dies qualifiziert als nicht dem Originaltext zugehörig und den „eigenen gedanken“ schlussfolgert, ihn als nicht falsch bestimmt (Z. 64). In Zeile 69 elizitiert die Lehrperson die Autorschaft, indem sie direkt nach individuellen Lösungen für das konkrete Schreibproblem - Wie mache ich im Exzerpt eigene und fremde Gedanken deutlich? - fragt. Aufgerufen wird also hypothetische Autorschaft und nicht reale, die in der direkten Frage nach den individuellen Realisationen in den Texten bestehen könnte. S7 setzt in Zeile 71 jedoch die eigene Autorschaft relevant und berichtet darüber, wie er*sie konkret dieses Handlungsproblem gelöst hat, was von der Lehrperson auch ratifiziert wird.

Funktional wird deutlich, dass solche überaus häufig genutzten Szenarien der Erweiterung von Varianz dienen und potenzielle Möglichkeiten offerieren, wie eine textuelle Anforderung gelöst werden kann. Diese Feedbackpraktiken rücken in die Richtung kleinräumiger, aktiver, schreibdidak- 


\begin{tabular}{|c|c|c|}
\hline 059 & LP & $\begin{array}{l}{ }^{\circ} \mathrm{h} \text { ah wo sehen sie da eigene geDANken? } \\
(0,7)\end{array}$ \\
\hline 060 & S7 & ahm zum beispiel \\
\hline 061 & & $\begin{array}{l}<<\text { liest vor }>\text { einige unserer vorstellungen haben doch mit } \\
\text { wissenschaftlich arbeiten nichts gemein, > }\end{array}$ \\
\hline 062 & & also so stehts jetzt nicht im text DRINnen \\
\hline 063 & & das ist so ein eigener geDANke \\
\hline 064 & & was [wahrscheinlich auch STIMmt; ] \\
\hline 065 & LP & [das stimmt (.) ja ] \\
\hline 066 & S7 & aber halt jetzt nicht vom text $(+++)$ \\
\hline 067 & LP & $\mathrm{hm} \_\mathrm{hm}(0,2) \mathrm{hm} \_\mathrm{hm}$ \\
\hline 068 & & $(5,5)$ \\
\hline 069 & LP & $\begin{array}{l}\text { wie würden sie das MAchen (.) dass (.) ahm } \\
(2,2)\end{array}$ \\
\hline 070 & & $\begin{array}{l}\text { den eigenen geDANken FOLgen- } \\
(0,8)\end{array}$ \\
\hline 071 & S7 & $\begin{array}{l}\text { also I: hab eigentlich haha <<lachend }>\text { beim Eigenen } \\
\text { exzerpt }>{ }^{\circ} \text { h DRUNter und drüber so halt in kurSIV meine } \\
\text { gedanken geschrieben; }\end{array}$ \\
\hline 072 & & damit ma erkennt was meine ge[danken] so sind \\
\hline 073 & LP & {$\left[\mathrm{hm} \_\mathrm{hm}\right]$} \\
\hline & & geNAU \\
\hline
\end{tabular}

Abb. 15 Aus: schr_2911 (00:09:51-00:10:23)

tischer Feedbackintervention; sie zeigen die Verknüpfung der Aufgaben „Probleme klären“ und „mögliche Überarbeitung ableiten“, wie sie Grieshammer (2018, S. 89) für Gespräche in der Schreibberatung herausarbeitet.

Als Feedbackintervention im Auswertungsgespräch zeigt sich der Umgang mit Autorschaft als geradezu paradoxe Intervention, die dennoch viele Möglichkeiten bereithält. So gestattet das umfangreiche Spektrum der Herstellung von Bezugnahmen auf das konkrete Auswertungsobjekt, flankiert von analytischen Schwerpunktsetzungen, präzise Arbeit mit epistemischem Gewinn auf der Ebene von Fachlichkeit. In didaktischer Perspektive wird deutlich, dass es offenbar nicht leicht möglich zu sein scheint, sich selbst, den eigenen Schreibprozess und dessen Produkt gerade nicht in Beziehung zu den Auswertungsfragen zu setzen, wenn dem Auswertungsgespräch doch eine eigene Schreibaktivität vorausging. An den Feedbackpraktiken im Umgang mit der Autorschaft zeigt sich also, dass Studierende hier konstruktiv ihren eigenen Lernerfolg bzw. die Nachhaltigkeit der gemeinsamen Arbeit sichern, wenn sie sich doch als Autor*innen zeigen. Sichtbar eigene Verantwortung für den Text zu übernehmen und sich einem Gespräch darüber zu stellen, könnte die Möglichkeiten der Interventionsformen von Feedback im Sinne der Kompetenzentwicklung erweitern. Es unterläuft aber gleichzeitig den verallgemeinernden und damit Wissen konstituierenden Ansatz, der der Anonymisierung zugrunde liegt. Nichtsdestotrotz ist es notwendig, über die Bedingungen der
Beteiligungsweise der Referenzperson im Auswertungsgespräch als ein Problem der Autorschaft nachzudenken, was von Sieber (2018) als Desiderat der Schreibdidaktik benannt wird.

\section{Fazit}

Es zeigt sich, dass solche verhältnismäßig neuen Gegenstände wie die Entwicklung und Optimierung von professionsorientierter Schreibkompetenz in der Hochschullehre durch die Etablierung neuer Interaktionstypen die Grenzen zwischen Hochschullehre und Coaching durchlässiger werden lassen, da sie gemeinsame Bezugspunkte haben.

Zum einen ist das die Orientierung am Individuum, dessen individuelle Handlungsfähigkeit (wieder-)hergestellt bzw. erweitert werden soll. In den Auswertungsgesprächen ist die aktive Involvierung der Schreibenden in den Arbeitsprozess die dafür wesentliche Größe und der sukzessive Ausbau der Abstraktion vom praktischen Tun hin zu verallgemeinernder, regelerkennender Distanz dient darüber hinaus dem Erreichen konkreter bildungsbezogener Ziele wie der Förderung von Analyse- und Reflexionskompetenz sowie dem Aufbau von (Fach-)Wissen. Die Orientierung am Problem ist in diese Prozesse eingelagert.

Zum anderen ist es der kommunikative Prozess. Lehre wie Schreibcoaching vollziehen sich im und durch das Gespräch, hier als Auswertungsgespräch, und in den Feed- 
backprozessen wird Erkenntnis im und durch das gemeinsame Gespräch generiert. Daher werden Interaktivität und Prozessualität wichtig, es zeigt sich geteilte Handlungstypik aufgrund geteilter kommunikativer Aufgaben sowie geteilte kommunikative Aktivitäten, die jedoch lokal und kontextualisiert etabliert werden. Des Weiteren ist die Rolle der*des Coachs sowie der Lehrperson mit ähnlichen Steuerungsaufgaben verbunden. Dazu gehören die Aufgaben der Phasierung des Gesprächs hinsichtlich der Eröffnung und den darin notwendigen Zügen, eine spezifische Coaching- bzw. Auswertungssituation zu konstituieren. Außerdem gehört es zum kommunikativen Handeln der*des Coachs, ergebnisoffen und ohne Direktiven den Coachingprozess zu strukturieren, Beziehung zu gestalten, lösungsorientiert zu arbeiten und Klient*innen zur Reflexion anzuregen. Das ähnelt den Steuerungsaufgaben der Lehrpersonen und zeigt sich an konkreten kommunikativen Entscheidungen wie dem Weglassen bestimmter typischer Interventionen aus der Lehre wie z.B. der Korrektur von Fachtermini, um die Orientierung auf den Feedbackprozess aufrecht zu halten. Hier hat die Lehrpraxis systematische Praktiken ausgebildet, um Reflexionsprozesse produktiv, wissensbasiert und vor allem konkret gegenstandsbezogen anzuregen.

Eine gemeinsame Klammer kann der Veränderungsbegriff sein (Graf et al. 2019). Die Ko-Konstruktion von Veränderung stellt sich in der Lehr-Lern-Situation als mehrfache Anforderung: als Veränderung am Text, als Veränderung der Analysekompetenz und ganz individuell als Veränderung des Autors/der Autorin und des konkreten Schreibprozesses. Veränderung zeigt sich hier zunächst nicht ausgehend von spezifischen individuellen Kommunikationsproblemen als vielmehr als eine professionsorientiert gebundene Veränderung eigener Schreibkompetenz. Es handelt sich damit um eine ziel- und zweckgerichtete Veränderung, deren Richtung von den curricular definierten Bildungszielen erst einmal vorgegeben sind und bei denen die Lehrperson sich verorten muss bzw. den Weg dahin gestalten muss.

Die Ergebnisse haben Implikationen auf mehreren Ebenen. Erstens können die zugrundeliegenden Interaktionsanalysen authentischer Feedbackprozesse in Auswertungsgesprächen die empirische Kontrastfolie liefern, mit der andere, ähnliche Interaktionstypen verglichen werden können; zugleich kann die interaktionale Coachingprozessforschung erweitert werden, indem ein spezifischer Lehr-Lern-Kontext einbezogen wird. Zweitens erlauben die Mikroanalysen, aus den rekonstruierten Praktiken Good-Practice-Beispiele für Feedback sowie für die produktive Gestaltung von Auswertungsprozessen herauszuarbeiten. Das wiederum kann das Repertoire von Schreibcoachs hinsichtlich der Varianz von Feedbackpraktiken erweitern. Drittens können aus den vorliegenden Analysen Gelingensbedingungen abgeleitet werden, die es erlauben, traditionelle Feedbackformen zu modi- fizieren und Feedbackgestaltung zu klären. Hervorgehoben wird in der Literatur der hohe Stellenwert des Verstehens von Feedback, da es als Garant für Effektivität gilt. Dafür müssen Feedbackprozesse jedoch aktiv gestaltet und als eigenständige Leistung mit einem spezifischen Ergebnis angesehen werden, was beispielsweise vom Instrument der Textkommentierungen als Feedback (Knorr 2012a) nicht geleistet wird. Um diese Gelingensbedingungen zu erkennen, könnte die empirische Schreibforschung verstärkt auf interaktionszentrierte Ansätze des Coachings zurückgreifen und diese als Analysekategorien nutzbar machen.

Zuvorderst zeigen die empirischen Daten aber, dass Interdisziplinarität für Forschung sowie Weiterentwicklung von Coaching, Hochschullehre oder Schreibwissenschaft unumgänglich ist. Denn die reale Textfeedback-Situation hält sich nicht an disziplinäre Grenzen.

Danksagung Ich bedanke mich herzlich bei Thomas Spranz-Fogasy, der mich bestärkt hat, die ersten Ideen zur Verbindung von Coaching und Lehr-Lern-Settings in interaktionsanalytischer Hinsicht auszuarbeiten und mir konstruktive Hinweise dazu gegeben hat. Bei meiner Innsbrucker Kollegin Bernadette Rieder sowie bei den anonymen Gutachter*innen möchte ich mich für kritische Lektüren der Vorfassung dieses Beitrags bedanken, die maßgeblich zur Verbesserung des Textes beigetragen haben.

Open Access Dieser Artikel wird unter der Creative Commons Namensnennung 4.0 International Lizenz veröffentlicht, welche die Nutzung, Vervielfältigung, Bearbeitung, Verbreitung und Wiedergabe in jeglichem Medium und Format erlaubt, sofern Sie den/die ursprünglichen Autor(en) und die Quelle ordnungsgemäß nennen, einen Link zur Creative Commons Lizenz beifügen und angeben, ob Änderungen vorgenommen wurden.

Die in diesem Artikel enthaltenen Bilder und sonstiges Drittmaterial unterliegen ebenfalls der genannten Creative Commons Lizenz, sofern sich aus der Abbildungslegende nichts anderes ergibt. Sofern das betreffende Material nicht unter der genannten Creative Commons Lizenz steht und die betreffende Handlung nicht nach gesetzlichen Vorschriften erlaubt ist, ist für die oben aufgeführten Weiterverwendungen des Materials die Einwilligung des jeweiligen Rechteinhabers einzuholen.

Weitere Details zur Lizenz entnehmen Sie bitte der Lizenzinformation auf http://creativecommons.org/licenses/by/4.0/deed.de.

\section{Literatur}

Bachmann, T., \& Becker-Mrotzek, M. (2010). Schreibaufgaben situieren und profilieren. In T. Pohl \& T. Steinhoff (Hrsg.), Textformen als Lernformen. (S. 191-209). Duisburg: Gilles \& Francke.

Baurmann, J. (2014). Prozessorientierung und Methoden des Schreibunterrichts. In H. Feilke \& T. Pohl (Hrsg.), Schriftlicher Sprachgebrauch: Texte verfassen (S. 349-363). Baltmannsweiler: Schneider Verlag Hohengehren.

Becker-Mrotzek, M., \& Böttcher, I. (2014). Schreibkompetenz entwickeln und beurteilen: Praxishandbuch für die Sekundarstufe I und II (5. Aufl.). Berlin: Cornelsen Scriptor.

Bloxham, S. (2013). Building, standards' frameworks. The role of guidance and feedback in supporting the achievement of learners. In S. Merry, M. Price, D. Carless \& M. Taras (Hrsg.), Reconceptualising feedback in higher education.: developing dialogue with students (S. 64-74). New York: Routledge. 
Brown, R. (2010). Representing audiences in writing center consultation: a discourse analysis. The Writing Center Journal, 30(2), $72-99$.

Dainton, N. (2018). Feedback in der Hochschullehre. Bielefeld: utb.

Dengscherz, S. (2019). Professionelles Schreiben in mehreren Sprachen: Strategien, Routinen und Sprachen im Schreibprozess. Berlin u.a.: Peter Lang. https://doi.org/10.3726/b16495.

Deplazes, S., Graf, E.-M., \& Künzli, H. (2018). Das TSSP-Modell Eine Blaupause für die Coaching-Prozessforschung. Coaching । Theorie \& Praxis, 4(1), 69-82.

Deppermann, A. (2008). Gespräche analysieren: Eine Einführung. Wiesbaden: VS.

Deppermann, A. (2013). Analytikerwissen, Teilnehmerwissen und soziale Wirklichkeit in der ethnographischen Gesprächsanalyse. In M. Hartung \& A. Deppermann (Hrsg.), Gesprochenes und Geschriebenes im Wandel der Zeit. Festschrift für Johannes Schwitalla (S. 32-59). Mannheim: Verlag für Gesprächsforschung.

Deppermann, A. (2018). Sprache in der multimodalen Interaktion. In A. Deppermann \& S. Reineke (Hrsg.), Sprache im kommunikativen, interaktiven und kulturellen Kontext. (S. 51-85). Berlin u.a.: De Gruyter.

Deppermann, A., \& Schmitt, R. (2007). Koordination: Zur Begründung eines neuen Forschungsgegenstandes. In R. Schmitt (Hrsg.), Koordination. Analysen zur multimodalen Interaktion (S. 15-54). Tübingen: Narr.

Deppermann, A., Feilke, H., \& Linke, A. (2016). Sprachliche und kommunikative Praktiken: Eine Annäherung aus linguistischer Sicht. In A. Deppermann, H. Feilke \& A. Linke (Hrsg.), Sprachliche und kommunikative Praktiken (S. 1-23). Berlin u.a.: De Gruyter.

Frank, A., Haacke, S., \& Lahm, S. (2007). Schlüsselkompetenzen: Schreiben in Studium und Beruf. Stuttgart: J. B. Metzler.

Graf, E.-M. (2015). Kommunikative Basisaktivitäten im CoachingGespräch: Ein linguistischer Beitrag zur Coaching-Prozessforschung. Coaching | Theorie \& Praxis, 1(1), 5-14.

Graf, E.-M., Aksu, Y., Pick, I., \& Rettinger, S. (Hrsg.). (2011). Beratung, Coaching, Supervision.: Multidisziplinäre Perspektiven vernetz.t. Wiesbaden: VS.

Graf, E.-M., Scarvaglieri, C., \& Spranz-Fogasy, T. (2019). Pragmatik der Veränderung in helfenden Berufen - Einführung. In E.M. Graf, C. Scarvaglieri \& T. Spranz-Fogasy (Hrsg.), Pragmatik der Veränderung: Problem- und lösungsorientierte Kommunikation in helfenden Berufen. Tübingen: Narr.

Grieshammer, E. (2017). Gesprächsanalyse als Methode der Schreibforschung. In M. Brinkschulte \& D. Kreitz (Hrsg.), Qualitative Methoden in der Schreibforschung. (S. 249-268). Bielefeld: wbv.

Grieshammer, E., Liebetanz, F., Peters, N., \& Zegenhagen, J. (2013). Zukunftsmodell Schreibberatung: Eine Anleitung zur Begleitung von Schreibenden im Studium (2. Aufl.). Baltmannsweiler: Schneider Verlag Hohengehren.

Grieshammer, E. (2018). Textentwürfe besprechen: Analysen aus der akademischen Schreibberatung. Bielefeld: wbv.

Hartung, S. (2017). Lernförderliches Feedback in der Online-Lehre gestalten. In H. R. Griesehop \& E. Bauer (Hrsg.), Lehren und Lernen online: Lehr- und Lernerfahrungen im Kontext akademischer Online-Lehre (S. 199-217). Berlin Heidelberg: Springer VS.

Hattie, J., \& Timperley, H. (2007). The power of feedback. Review of Educational Research, 77(1), 81-112.

Jautz, S. (2018). In the Wind of Change: Wie Veränderungen im Coaching angestoßen werden können. Coaching I Theorie \& Praxis, $4(1), 33-44$.

Kluger, A. N., \& DeNisi, A. (1996). The effects of feedback interventions on performance: a historical review, a meta-analysis, and a preliminary feedback intervention theory. Psychological Bulletin, 119(2), 254-284.

Knorr, D. (2012a). Textkommentierungen. Formen und Funktionen. In D. Knorr \& A. Verhein-Jarren (Hrsg.), Schreiben unter Bedingungen von Mehrsprachigkeit. (S. 75-98). Berlin u.a.: Peter Lang.
Knorr, D. (2012b). Textüberarbeitungsprozesse durch Randkommentare initiieren. In U. Preußer \& N. Sennewald (Hrsg.), Literale Kompetenzentwicklung an der Hochschule (S. 245-264). Frankfurt a.M. u.a.: Peter Lang.

Kruse, O., \& Chitez, M. (2012). Schreibkompetenz im Studium: Komponenten, Modelle und Assessment. In U. Preußer \& N. Sennewald (Hrsg.), Literale Kompetenzentwicklung an der Hochschule (S. 57-83). Frankfurt a.M. u.a.: Peter Lang.

Kruse, O., \& Ruhmann, G. (2006). Prozessorientierte Schreibdidaktik: Eine Einführung. In O. Kruse, K. Berger \& M. Ulmi (Hrsg.), Prozessorientierte Schreibdidaktik (S. 13-35). Bern: Haupt.

Lave, J., \& Wenger, E. (1991). Situated learning: Legitimate peripheral participation. Cambridge: Cambridge University Press.

Lehnen, K. (2011). Wie sich das Schreiben für die Sprachreflexion in der Schule nutzen lässt - und umgekehrt. Ansätze einer reflexiven Schreibdidaktik. In B. Arendt \& J. Kiesendahl (Hrsg.), Sprachkritik in der Schule (S. 141-163). Göttingen: Vandenhoeck \& Ruprecht.

Mondada, L., \& Schmitt, R. (2010). Zur Multimodalität von Situationseröffnungen. In L. Mondada \& R. Schmitt (Hrsg.), Situationseröffnungen: Zur multimodalen Herstellung fokussierter Interaktion (S. 7-52). Tübingen: Narr.

Mondada, L. (2016). Conventions for multimodal transcription. Universität Basel. https://franzoesistik.philhist.unibas.ch/fileadmin/ user_upload/franzoesistik/mondada_multimodal_conventions. pdf. Zugegriffen: 18.01.2019.

Mönnich, A. (2010). Funktionen von Hörerrückmeldungen in Feedbackgesprächen. In N. Hinrichs \& A. Limburg (Hrsg.), Gedankenstriche - Reflexionen über Sprache als Ressource (S. 231-249). Tübingen: Stauffenburg.

Nussbaumer, M., \& Sieber, P. (1995). Über Textqualitäten reden lernen - z. B. anhand des „Zürcher Textanalyserasters“. Diskussion Deutsch, 26(141), 36-52.

Paul, I. (1999). Praktische Sprachreflexion. Tübingen: Niemeyer.

Pick, I. (2017). Zusammenführung der Beiträge: Entwicklung einer Typologie des Handlungstyps Beraten. In I. Pick (Hrsg.), Beraten in Interaktion.: Eine gesprächslinguistische Typologie des Beratens. (S. 427-470). Frankfurt a.M.: Peter Lang.

Pohl, T., \& Steinhoff, T. (2010). Textformen als Lernformen. In T. Pohl \& T. Steinhoff (Hrsg.), Textformen als Lernformen. (S. 5-26). Duisburg: Gilles \& Francke.

Rädiker, S., \& Kuckartz, U. (2019). Analyse qualitativer Daten mit MAXQDA: Text, Audio und Video. Wiesbaden: Springer VS.

Rettinger, S. (2011). Das Coaching-Erstgespräch: „Handlungsidentitäten“ in der Beziehungsgestaltung zwischen Coach und Klient. In E.-M. Graf In, Y. Aksu, I. Pick \& S. Rettinger (Hrsg.), Beratung, Coaching, Supervision: Multidisziplinäre Perspektiven vernetz.t (S. 149-166). Wiesbaden: VS.

Ruhmann, G., \& Kruse, O. (2014). Prozessorientierte Schreibdidaktik: Grundlagen, Arbeitsformen, Perspektiven. In S. Dreyfürst \& N. Sennewald (Hrsg.), Schreiben. Grundlagentexte zur Theorie, Didaktik und Beratung (S. 15-34). Opladen, Toronto: Barbara Budrich.

Sadler, R.D. (2010). Beyond feedback: developing student capability in complex appraisal. Assessment \& Evaluation in Higher Education, 35(5), 535-550.

Sadler, R.D. (2013). Opening up feedback. Teaching learners to see. In S. Merry, M. Price, D. Carless \& M. Taras (Hrsg.), Reconceptualising feedback in higher education.: developing dialogue with students (S. 54-63). New York: Routledge.

Schmölzer-Eibinger, S., Bushati, B., Ebner, C., \& Niederdorfer, L. (Hrsg.). (2018). Wissenschaftliches Schreiben lehren und lernen: Diagnose und Förderung wissenschaftlicher Textkompetenz in Schule und Universität. Münster: Waxmann.

Schwarze, C. (2015). Angemessenheitsverhandlungen in Auswertungsgesprächen in der Hochschullehre. Aptum. Zeitschrift für Sprachkritik und Sprachkultur, 11(02), 190-199. 
Schwarze, C., \& Rieder, B. (2014). Erkenntnisse über Sprache durch reflexionsorientierte Schreibdidaktik. Grundsätze und methodisch-didaktische Implikationen. In W. Hackl, K. Kupczyńska \& W. Wiesmüller (Hrsg.), Sprache - Literatur - Erkenntnis (S. 445-460). Wien: Praesens.

Selting, M., \& Auer, P., et al. (2009). Gesprächsanalytisches Transkriptionssystem 2 (GAT 2). Gesprächsforschung - OnlineZeitschrift zur verbalen Interaktion, 10, 353-402. http://www. gespraechsforschung-ozs.de/heft2009/px-gat2.pdf. Zugegriffen: 03.12.2020.

Sieber, P. (2008). Kriterien der Textbewertung am Beispiel Parlando. In: N. Janich (Hrsg.), Textlinguistik. 15 Einführungen (S. 271-289). Tübingen: Narr.
Sieber, P. (2018). Autorenschaft und Ästhetik - Abwesende in der Schreibdidaktik? Zeitschrift Schreiben, 9. Juli 2018, 1-10. www. zeitschrift-schreiben.eu/2018/\#sieber.

Steinhoff, T. (2007). Wissenschaftliche Textkompetenz: Sprachgebrauch und Schreibentwicklung in wissenschaftlichen Texten von Studenten und Experten. Tübingen: Niemeyer.

Publisher's Note Springer Nature remains neutral with regard to jurisdictional claims in published maps and institutional affiliations. 